\title{
Bayesian state-space modelling of the De Lury depletion model: strengths and limitations of the method, and application to the Moroccan octopus fishery
}

\author{
Marianne Robert, Abdelmalek Faraj, Murdoch K. McAllister and Etienne Rivot
}

Robert, M., Faraj, A., McAllister, M. K., and Rivot, E. 2010. Bayesian state-space modelling of the De Lury depletion model: strengths and limitations of the method, and application to the Moroccan octopus fishery. - ICES Journal of Marine Science, 67: 000-000.

The strengths and limitations of a Bayesian state-space modelling framework are investigated for a De Lury depletion model that accommodates two recruitment pulses per year. The framework was applied to the Moroccan fishery for common octopus (Octopus vulgaris) between 1982 and 2002. To allow identifiability, natural mortality $(M)$ and the recruitment rhythm were fixed, and the variance of both process and observation errors were assumed to be equal. A simulation-estimation (SE) approach was derived to test the performance of the method. If the data showed responses to harvest, the estimates of the most important figures, i.e. the initial abundance and the second recruitment pulse, were accurate, with relatively small bias. Results confirm that greater depletion yields smaller bias and uncertainty and that inferences are sensitive to the mis-specification of $M$. The 21 depletion series in the Moroccan dataset were jointly treated in a hierarchical model including random walk to capture the systematic fluctuations in estimates of catchability and initial abundance. The model provides estimates of the annual recruitment and monthly octopus population size. The recruitment estimates could be used to investigate the link between recruitment variability and the coastal North African upwelling regime to improve understanding of the dynamics and management of octopus stocks.

Keywords: Bayesian, De Lury depletion model, hierarchical model, MCMC, octopus, recruitment, state-space modelling.

Received 26 June 2009; accepted 8 February 2010.

M. Robert and E. Rivot: UMR 985 UMR ESE Agrocampus OUEST, INRA, Ecologie et Santé des Ecosystèmes, Agrocampus OUEST, laboratoire Ecologie Halieutique, 65 rue de St-Brieuc, CS 84 215, 35042 Rennes cedex, France. M. Robert: IRD, Seychelles BP 570 Victoria, Mahe, Seychelles. A. Faraj: Laboratoire Approches et Méthodologie, INRH, 2 rue de Tiznit, Casablanca, Morocco. M. K. McAllister: Fisheries Centre, Aquatic Ecosystems Research Laboratory, University of British Columbia, 2202 Main Mall, Vancouver, BC, Canada V6T 1Z4. Correspondence to E. Rivot: tel: +33 2 234859 34; fax: +332234855 35; e-mail: etienne.rivot@agrocampus-ouest.fr.

\section{Introduction}

Cephalopods are of increasing commercial fisheries importance worldwide (Caddy and Rodhouse, 1998; Payne et al., 2006). Most commercially important cephalopod species have a short life cycle, grow rapidly, and are semelparous. Catches show large year-on-year fluctuations driven by the influence of environmental conditions on recruitment (Agnew et al., 2000; Faure et al., 2000; Otero et al., 2008; Pierce et al., 2008). Owing to poor knowledge of their biology and ecology and to their short lifespan, the assessment of cephalopod stocks requires specialized methodology. As age determination is difficult, biomass surplus production models are often used for stock assessments (FAO, 2006). However, providing management advice based on the results from annual surplus production models may not be the most suitable approach for managing cephalopod fisheries. Indeed, few generations overlap, stocks respond at fine time-scales to fluctuations in the environment and fishing pressure, and within-season advice is often needed (Pierce and Guerra, 1994; Payne et al., 2006).

Several authors have suggested that stock assessment of such short-lived species should combine long- or medium-term assessment methods (e.g. annual biomass surplus production models) with short-term in-season assessments based on models running on shorter (typically monthly or weekly) time-steps (Pierce and Guerra, 1994; Agnew et al., 1998; Roa-Ureta and Arkhipkin, 2007). Depletion models (Leslie and Davis, 1939; De Lury, 1947) are good candidates for in-season monitoring and management of cephalopod fisheries. Intense rates of exploitation generally induce sharp depletions in abundance after the main recruitment within a year. The depletion model is designed to capture such within-season dynamics and can be used to track in real time the depletions in abundance under fishing pressure (Pierce and Guerra, 1994; Agnew et al., 1998; Young et al., 2004), allowing in-season adaptations of management measures. Forecasts under alternative scenarios of fishing effort can also be derived, so allowing evaluation of whether the initial potential of the stock was under- or over-appraised, and adjustment of effort and catches for the end of the fishing season. Depletion models have been applied successfully in the assessment of the squid stock of the Falkland Islands within a frequentist statistical approach (Beddington et al., 1990; Rosenberg et al., 1990; Agnew et al., 1998; Roa-Ureta and Arkhipkin, 2007) and a Bayesian approach (McAllister et al., 2004). Royer et al. (2002) used a depletion model to study squid recruitment in the English Channel. Depletion models have also been used to describe the dynamics 
of other exploited species, such as Cuban shelf spiny lobster (González-Yáñez et al., 2006).

Although process noise and observation errors are not uncommon in fisheries models, the depletion model has never been considered in a state-space modelling (SSM) framework accounting for both sources of errors. This work investigates the strengths and limitations of a Bayesian SSM (BSSM) framework for the De Lury depletion model, with an application to the Moroccan fisheries of common octopus (Octopus vulgaris) between 1982 and 2002. We offer three substantial new contributions. First, as far as we can ascertain, the work provides the first BSSM approach of the De Lury depletion model including both process and observation errors and an estimation of a secondary recruitment pulse. Second, extensive evaluation of the sensitivity of a Bayesian version of this model to alternative model forms and data configurations is provided. Third, the hierarchical Bayesian approach of McAllister et al. (2004) was extended by introducing a random-walk formulation to capture the systematic fluctuations of the time-series of initial abundance and catchability.

A vast continental shelf combined with the large and permanent Canarian upwelling system makes NW African waters one of the world's richest fishing grounds (Balguerías et al., 2000). Octopus fisheries are of great social and economic importance in the region, especially in Morocco (Faraj and Bez, 2007). Moroccan catches attributed to O. vulgaris have shown marked fluctuations, with maxima in 1991 and 2000 (around $110000 \mathrm{t}$ ) and minima in 1997 and 2003 (50 000 and 25000 t, respectively; FAO, 2006). Stock assessments, mostly based on surplus production models, reveal that octopus stocks in the region have been overexploited since the end of the 1980s (FAO, 2006). The depletion model has recently been advocated as a method of choice to be implemented in the region (Faraj et al., 2006), because it allows conducting of in-season stock assessment and management. A depletion model was developed for the Moroccan case study. Octopus along the Moroccan coast show two recruitment pulses per year (Balguerías et al., 2002; Faraj and Bez, 2007). The main recruitment is in autumn and catch rates peak around November. A secondary recruitment pulse appears during spring. Hence, the depletion model was extended to include the spring recruitment (Royer et al., 2002; González-Yáñes et al., 2006).

The depletion model was developed within a Bayesian framework because that provides a flexible rationale for stochastic statistical modelling, inferences, and predictions (Ellison, 2004; Clark, 2005). Since the 1990s, the Bayesian framework has been widely applied in fisheries sciences (Walters and Ludwig, 1994; Punt and Hilborn, 1997; McAllister and Kirkwood, 1998; Harwood and Stokes, 2003). It allows the integration of different types of information (Hilborn and Lierman, 1998) and multiple sources of uncertainty in data and models. Diagnostics and predictions are derived using probabilistic rationale, and results are easily embedded within a formal decision analysis. In particular, BSSM coupled with sequential Monte Carlo methods such as Markov Chain Monte Carlo (MCMC) simulations have proved able to handle discrete-time dynamic models with process error stemming from uncertainty in the dynamics of the system and measurement and sampling errors in the data (Meyer and Millar, 1999; Millar and Meyer, 2000; Buckland et al., 2004; Rivot et al., 2004; Thomas et al., 2005; Swain et al., 2009).

A simulation-estimation (SE) analysis was conducted to assess the strengths and limitations of the approach. Even if the methodology is technically well established, deriving Bayesian inferences on a SSM is a non-trivial task. Fitting models to simulated data helps to determine the performance of the method, because the true values of the parameters used in the simulation are known. SE approaches have been widely used in the frequentist framework (for recent examples, see Punt, 2003; Magnusson and Hilborn, 2007; Trenkel, 2008). The analyses are less immediate and more laborious in the Bayesian framework, and examples are rarer (see Hammond and Trenkel, 2005; Tang and Heron, 2008; Zhang et al., 2009, for recent examples). In the current work, special attention is devoted to the SE approach designed to assess the performance of the estimation method under different data and model configurations.

The framework was applied to analyse catches and catch per unit effort (cpue) data from the Moroccan octopus fishery between 1982 and 2003. The 21 depletion series were jointly treated in a hierarchical model. Hierarchical Bayesian models have been advocated as appropriate methods to analyse jointly a population of similar datasets that share common characteristics (Rivot and Prévost, 2002; Gelman et al., 2004; McAllister et al., 2004; Rivot et al., 2008). They offer a probabilistic structure to model the cross-population variability in parameters that underlies different datasets and to derive posterior predictive distributions for the parameters of a yet unsampled dataset. McAllister et al. (2004) applied Bayesian hierarchical modelling (BHM) to a set of historical depletion series for Falkland Islands squid. They show how hierarchical modelling probabilistically shares information among the different depletion series and improves the estimation of key parameters. The hierarchical structure they proposed was extended by introducing random walk (RW) to capture autocorrelated fluctuations in the time-series of initial abundance and catchability. Indeed, the values of $N_{1}$ are likely to show autocorrelated fluctuations because of the population dynamics and environmental influences. Fishing efficiency is also likely to show fluctuations caused by gradual changes in behaviour and technological improvements to the fishing fleet.

\section{Material and methods \\ Data}

The depletion model was used to mimic the decrease in abundance (in numbers) of octopus in Morocco for 21 depletion series between 1982 and 2003. For each series, the model was built on a monthly time-step. In Moroccan waters, the maximum abundance of octopus is observed in November, the opening of the fishery (Figure 1). Overall, there were 21 depletion series beginning in November 1982 (Figure 2). The data used in this work were collected by the Moroccan Institute for Fisheries Research (INRH) and the Moroccan Fisheries Department (Faraj et al., 2006), and the methods used to calculate monthly catches and cpue for the period 1982-2003 are presented below.

Total annual catches in Moroccan waters (Moroccan catches for the artisanal, coastal, and industrial fleets, and catches of the foreign industrial fleet) were available for the years 1982-2003. First, total annual catches were disaggregated to a monthly timestep, based on a survey of the industrial Moroccan fleet, because a survey of the other fleets did not provide the same level of information. Second, monthly catches by weight were converted to numbers via an average weight-at-month relationship. This relationship was established from samples taken from artisanal and coastal landings between 2001 and 2005. 


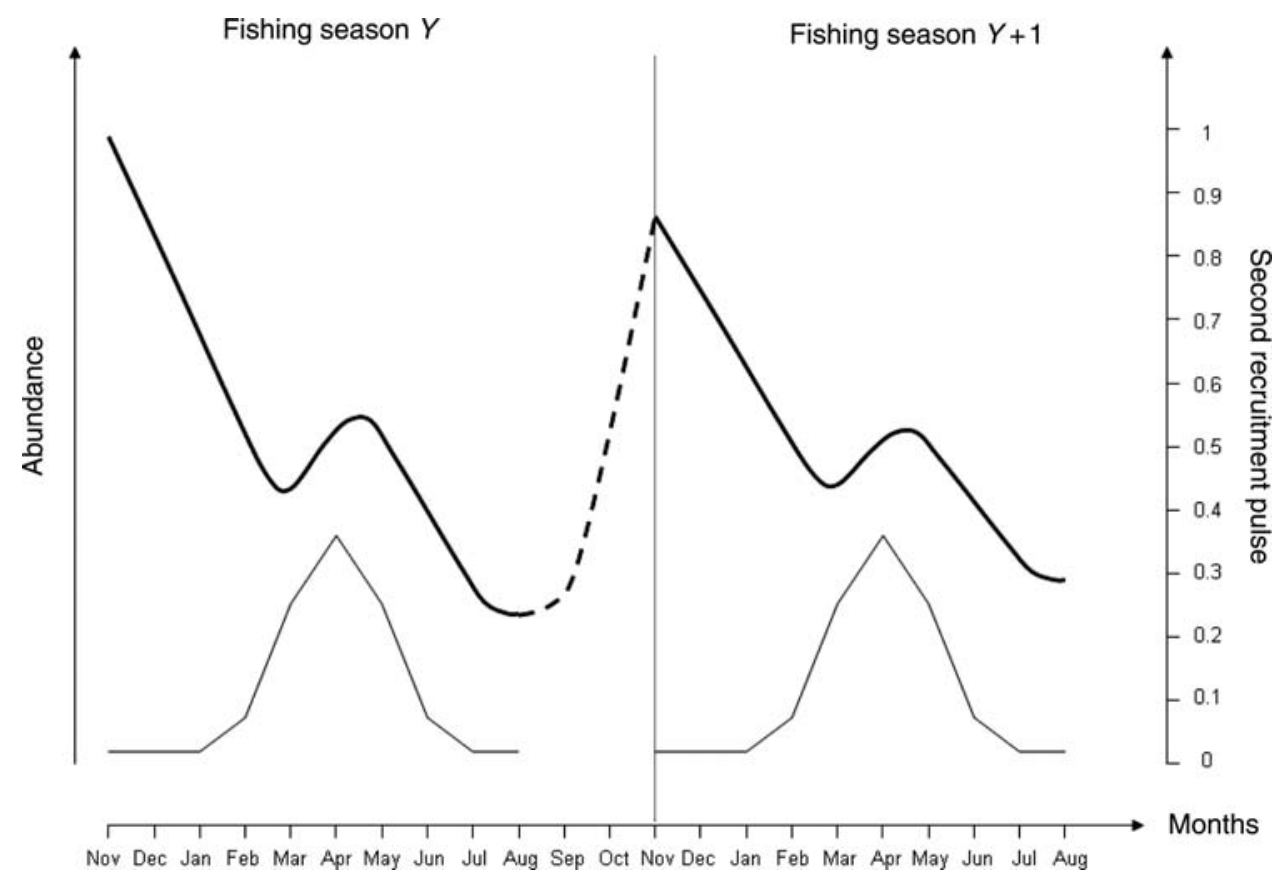

Figure 1. Schematic diagram of the shape of the abundance trajectory (arbitrary units) of octopus in Moroccan waters. The thick line depicts the typical shape (arbitrary units) of the abundance observed from abundance indices (solid line) and unobserved (dashed line). The main recruitment pulse is just before the opening of the fishing season, typically in November. The thin line depicts the proportion of the secondary recruitment $R$ that occurs each month; it takes place between February and June, with a peak in April (values are given in text).

The cpue series of the industrial Moroccan fleet were used. The cpue was calculated monthly by dividing the catches (in numbers as defined above) by the duration of the trip (in days at sea). From the 1982-2003 time-series of catches and cpue (monthly timestep), 21 depletion series were selected according to the typical shape of the abundance during one fishing season (Figure 1) and to a decision rule: for each season, the start of each depletion series (associated with the highest cpue value) was defined as the opening of the fishery, and the end of the depletion series was considered as the inflection point in the series of cpue just before the opening of the fishery season the following year (Figure 1). Roughly speaking, all depletion series start in November, but the duration of the series is not constant over the time-series (Table 1).

\section{BSSM of the depletion model}

\section{Dynamic equation}

Depletion models are based on a discrete-time sequential equation that mimics the decrease in the abundance (in numbers) of a closed population in response to removals (Rosenberg et al., 1990; Gonzalez Cano and Restrepo, 2001). Rosenberg et al. (1990) proposed a model that assumes that the abundance at timestep $t+1$ depends on the abundance at time $t$, the cumulative catches $c_{t}$ between $t$ and $t+1$, and natural mortality, $M$. The latter rate is assumed constant over time. The process is initialized by the initial abundance, $N_{1}$. Following Royer et al. (2002) or González-Yáñes et al. (2006), a secondary recruitment pulse for Moroccan octopus was accounted for by modifying the depletion model to incorporate a secondary recruitment, denoted $R$, within the fishing season [Equation (1)] with a certain rhythm. The recruitment rhythm is denoted $\left(\lambda_{1}, \ldots, \lambda_{n}\right)$, with $\lambda_{t}$ defined as the proportion of $R$ that takes place in month $t$ (and the values of $\lambda_{t}$ sum to 1$)$. The recruitment rhythm was considered known to ensure that all parameters can be estimated (Royer et al., 2002). According to Pope's approximation (Pope, 1972), we considered the cumulative catches $\left(c_{t}\right)$ and the recruitment $\lambda_{t} R$ as punctual events occurring exactly in the middle of the period $[t, t+1]$. Process errors were assumed to be independent and identically distributed (iid) multiplicative lognormal (Halley and Inchausti, 2002) with variance $\sigma_{\mathrm{p}}^{2}$ :

$$
\begin{aligned}
& N_{t+1}=\left(N_{t} \mathrm{e}^{-M}-c_{t} \mathrm{e}^{-M / 2}+\lambda_{t} \mathrm{Re}^{-M / 2}\right) \mathrm{e}^{\varepsilon_{t}} \\
& \text { with } \quad \varepsilon_{t} \stackrel{\text { iid }}{\sim} N\left(0, \sigma_{\mathrm{p}}^{2}\right) \quad \text { for } t=1, \ldots, n-1,
\end{aligned}
$$

where $N_{1}$ is the initial abundance.

The initial population size $N_{1}$, interpreted as the intensity of the main recruitment event, is in November (the opening of the fishery). Therefore, in our model, $t=1$ is in November of calendar year $a$. The secondary recruitment contributes to the fishery from spring to the end of summer (Figure 1) of calendar year $a+1$.

\section{Observation equation}

Estimation procedures rely on the fact that removing individuals from a population has a noticeable effect on some observable abundance index. A linear relationship is classically assumed between observed abundance indices $i_{t}$ and unobserved abundance $N_{t}$, with a scaling factor $q$ considered as constant over time. As $i_{t}$ is a cpue and because catches are assigned to the middle of the timestep, the abundance index is assumed to be proportional to the mean abundance between time-step $t$ and $t+1$. As for process errors, observation errors were assumed to be iid multiplicative 

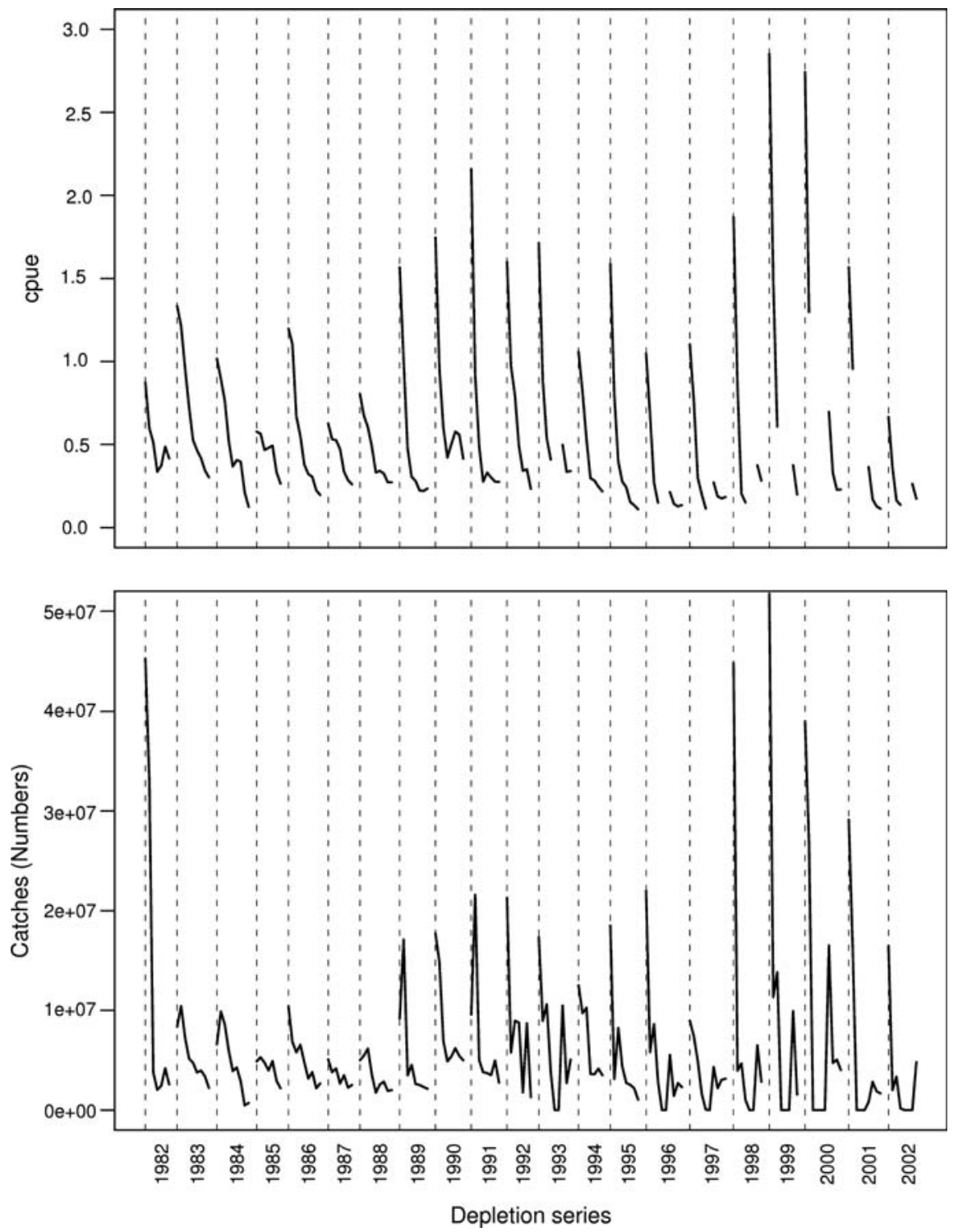

Figure 2. Total catches (in numbers) and abundance indices (cpue of the industrial fleet; thousands per day at sea) for the Moroccan fishery of octopus between 1982 and 2002. Catches and abundance indices are plotted on monthly time-steps for the 21 depletion series.

lognormal with variance $\sigma_{\mathrm{o}}^{2}$ :

$$
\begin{aligned}
i_{t}= & q\left(\frac{N_{t}+N_{t+1}}{2}\right) \mathrm{e}^{\omega_{t}} \\
& \text { with } \quad \omega_{t} \stackrel{\text { iid }}{\sim} N\left(0, \sigma_{\mathrm{o}}^{2}\right) \quad \text { for } t=1, \ldots, n-1 .
\end{aligned}
$$

The classical estimation approach relies on rewriting Equation (1) for each time-step $t$ (with $R=0$ and no process error) as a function of the initial population size $N_{1}$, and cumulative catches between $t=1$ and $t$. Then, substituting $N_{t}$ by $i_{t} / q$, and assuming an observation error on the abundance indices, the parameters $N_{1}$ and $q$ can be estimated by regressing abundance indices against cumulative catches [possibly in log-scale; Equation (3)]. The estimates of $q$ and $N_{1}$ are highly correlated because those parameters appear as a product in Equation (3). The performance of the estimation depends on the depletion intensity: the stronger the slope of the regression, the better the quality of the estimates (Agnew et al., 1998; McAllister et al., 2004; Magnusson and Hilborn, 2007):

$$
i_{t}=q N_{1} \mathrm{e}^{-(t-1) M}-q \sum_{j=1}^{j=t-1} c_{j} \mathrm{e}^{-(t-j-0.5) M} .
$$

Including recruitment $R$ and considering the model in an SSM framework with both process errors [Equation (1)] and observation errors [Equation (2)] markedly complicates the estimation. Process errors in the dynamic propagate across time, and the abundance indices $i_{t}$ can no longer be expressed as a simple function of $N_{1}$ and cumulative catches. Applying a Bayesian Monte Carlo approach for parameter estimation allows us to overcome this difficulty and to approximate the posterior distributions of all unknown variables in the model. 
Table 1. Dates of the start and the end of each of the 21 selected depletion series (start years 1982-2002).

\begin{tabular}{lll}
\hline Depletion series & Start & End \\
\hline 1 & November 1982 & April 1983 \\
2 & October 1983 & June 1984 \\
3 & October 1984 & June 1985 \\
4 & September 1985 & March 1986 \\
5 & October 1986 & June 1987 \\
6 & December 1987 & June 1988 \\
7 & October 1988 & June 1989 \\
8 & November 1989 & June 1990 \\
9 & November 1990 & June 1991 \\
10 & November 1991 & June 1992 \\
11 & October 1992 & April 1993 \\
12 & November 1993 & July 1994 \\
13 & November 1994 & May 1995 \\
14 & November 1995 & June 1996 \\
15 & November 1996 & August 1997 \\
16 & November 1997 & August 1998 \\
17 & November 1998 & June 1999 \\
18 & November 1999 & June 2000 \\
19 & November 2000 & August 2001 \\
20 & December 2001 & August 2002 \\
21 & December 2002 & July 2003 \\
\hline & &
\end{tabular}

\section{Fixed parameters}

To ensure that all parameters can be estimated, further hypotheses were formulated and several parameters were fixed according to the Moroccan case study. Process and observation error variances were set to be equal: $\sigma_{\mathrm{o}}^{2}=\sigma_{\mathrm{p}}^{2}=\sigma^{2}$. The magnitude of process and observation errors can seldom be specified a priori. In the absence of any information on $\sigma_{\mathrm{o}}^{2}$ and $\sigma_{\mathrm{p}}^{2}$, identifiability of the two variances is not guaranteed in SSM, and fixing the ratio of variances $\psi$ was needed to reduce the number of unknown parameters. Inferences in SSM are seldom sensitive to the value of $\psi$, unless it strongly favours observation or process error (Kimura et al., 1996; Schnute and Kronlund, 2002; Punt, 2003). Therefore, in the absence of any particular information on the relative magnitude of process vs. observation error when analysing real datasets, $\psi=1$ was used as a default choice.

The rate of natural mortality, $M$, was fixed at $0.1 \mathrm{month}^{-1}$ and considered to be constant. This corresponds to a survival rate of $<20 \%$ after 1.5 years for this short lifespan species.

The rhythm with which secondary recruitment occurs in the fishery was considered known. It was defined from previous studies (Balguerías et al., 2002) and knowledge from local experts (AF, pers. obs.). The secondary recruitment pulse was considered to take place between February and June, with a peak in April. The following values were considered: $\lambda=0.01,0.01$, $0.01,0.06,0.24,0.35,0.24,0.06,0.01$, and 0.01 (Figure 1).

\section{Priors}

The priors set on the parameters $N_{1}, R, q$ and the variance $\sigma^{2}$ in the baseline version of the model are defined in Table 2. Priors on $N_{1}$ and $R$ were defined as rather weak lognormal. For each depletion series, $R$ was defined a priori as a fraction $\alpha_{R}$ of $N_{1}$, with $\alpha_{R}$ (one for each depletion series) being drawn in a uniform $(0,1)$ prior distribution. This introduced the reasonable a priori constraint that the secondary recruitment $R$ is less than the primary recruitment $N_{1}$. The logarithm of the process error variance was given a
Table 2. Prior distributions for the four unknown parameters of the reference scenario of the SE approach (S1).

\begin{tabular}{lll}
\hline Parameter & Distribution & Parameter \\
\hline$N_{1}$ & $\sim$ Lognormal & Mode $=10^{8}, C V=2$ \\
$R$ & $R=\alpha_{R} N_{1}$ & $\alpha_{R} \sim$ uniform $(0,1)$ \\
$q$ & $\sim$ Lognormal & Mode $=10^{-7}, C V=2$ \\
$\sigma^{2}$ & $\log \left(\sigma^{2}\right) \sim$ uniform & $C V_{\min }=0.01, C V_{\max }=10 ;$ \\
& $\left(\log \left(\sigma_{\min }^{2}\right), \log \left(\sigma_{\max }^{2}\right)\right)$ & $\sigma^{2}=\log \left(C V^{2}+1\right)$ \\
\hline
\end{tabular}

Lognormal priors were specified in terms of the mode and the $\mathrm{CV}$ so as to better monitor their shape. $\sigma^{2}$ refers to the variance of the process and observation errors (supposed equal). The prior on the secondary

recruitment specifies that $R$ is a priori lower than the initial abundance $N_{1}$.

uniform prior with large bounds to mimic a non-informative prior in the sense of Jeffrey (Millar, 2002; Gelman et al., 2004).

\section{SE approach}

\section{Flow chart of the approach}

The performance of the estimation method was evaluated using simulated data. The flow chart of the SE approach has three steps.

Step 1: Simulation. Simulate abundance, catches, and abundance index trajectories according to an operating model with known parameters and randomness included in both process and observations. Each scenario first consisted of fixing a set of parameters $\left(M, q, R, N_{1},\left\{\lambda_{t}\right\}_{t=1, \ldots, n}, \sigma^{2}\right.$, and monthly harvest rates $\left.\left\{h_{t}\right\}_{t=1, \ldots, n}\right)$ which were used to simulate trajectories of abundance $\left\{N_{t}\right\}_{t=1, \ldots, n}$, catches $\left\{c_{t}\right\}_{t=1, \ldots, n}$, and abundance indices $\left\{i_{t}\right\}_{t=1, \ldots, n}$ following Equations (1) and (2). To remain consistent with Pope's approximation in Equation (1), catches were simulated as $c_{t}=h_{t} N_{t} \mathrm{e}^{-M / 2}$. The trajectories were simulated over $n=$ 10 time-steps, the maximum length (in months) of a fishing season in Morocco. To evaluate the average performance of the estimation method, 50 repetitions of the SE procedure were conducted for each scenario (see below). Preliminary analyses showed that 50 trials were sufficient to obtain a stabilized evaluation of the performance of the method.

Step 2: Bayesian estimation. Consider the simulated catches and abundance index trajectories as data and use the Bayesian framework to estimate the abundance trajectory and the underlying parameters. For each simulated dataset $\left\{i_{t}, c_{t}\right\}_{t=1, \ldots, n}$, a Bayesian estimation of unknown variables $\left(q, \sigma^{2}, R,\left\{N_{t}\right\}_{t=1, \ldots, n}\right)$ was performed. $M$ and $\left\{\lambda_{t}\right\}_{t=1, \ldots, n}$ were considered known in the estimation. Note that for almost all scenarios, the model used for the estimation was the same as the operating model used for simulating the data. This ensured that any differences between simulated and estimated quantities were attributable to the performance of the estimation method and not to model mis-specification. A model error, i.e. a difference between the operating model and the simulated model, was introduced only in a few scenarios to assess the sensitivity of the method to mis-specification of $M$ and $\lambda$ and to the hypothesis made on the error structure.

Step 3: Quantification of the quality of the estimations. The performance of the estimation method was evaluated with respect to how well the marginal posterior distribution of each parameter or unknown state variable estimated the true or simulated value. The evaluation uses three complementary criteria. First, the mean relative error (MRE) was calculated to quantify the bias of the Bayesian estimation. For each parameter $\theta$ and each simulation $s$ $(s=1, \ldots, 50)$, the relative error $\operatorname{RE}\left(\theta_{s}\right)$ was calculated following Equation (4a), with $E\left(\theta \mid\right.$ data $\left._{s}\right)$ the expected posterior mean of 
Table 3. Characteristics of the 16 scenarios tested in the SE approach.

\begin{tabular}{|c|c|c|c|c|c|c|c|c|c|c|}
\hline \multirow[t]{3}{*}{ Scenario } & \multicolumn{5}{|c|}{ Simulation } & \multicolumn{5}{|c|}{ Estimation } \\
\hline & \multirow[b]{2}{*}{ Depletion at 3 months } & \multirow[b]{2}{*}{$C V$} & \multirow[b]{2}{*}{$\lambda$} & \multirow[b]{2}{*}{$M$} & \multirow[b]{2}{*}{ Fishery closure } & \multicolumn{2}{|c|}{ Prior on $N_{1}$} & \multirow[t]{2}{*}{$M$} & \multirow[t]{2}{*}{ Missing data } & \multirow[t]{2}{*}{ Error structure } \\
\hline & & & & & & $C V$ & Mode & & & \\
\hline$\overline{\mathrm{S} 1}$ & High & 0.1 & Base & 0.1 & No & 2 & $10^{8}$ & 0.1 & No & SSM \\
\hline S2 & Low & 0.1 & Base & 0.1 & No & 2 & $10^{8}$ & 0.1 & No & SSM \\
\hline S3 & High & 0.1 & Base & 0.2 & No & 2 & $10^{8}$ & 0.2 & No & SSM \\
\hline S4 & High & 0.2 & Base & 0.1 & No & 2 & $10^{8}$ & 0.1 & No & SSM \\
\hline S5 & High & 0.3 & Base & 0.1 & No & 2 & $10^{8}$ & 0.1 & No & SSM \\
\hline S6 & High & 0.1 & Base & 0.1 & No & 5 & $10^{8}$ & 0.1 & No & SSM \\
\hline S7 & High & 0.1 & Base & 0.1 & No & 2 & $10^{7}$ & 0.1 & No & SSM \\
\hline S8 & High & 0.1 & Base & 0.1 & No & 2 & $10^{9}$ & 0.1 & No & SSM \\
\hline S9 & High & 0.1 & Base & 0.2 & No & 2 & $10^{8}$ & 0.1 & No & SSM \\
\hline S10 & High & 0.1 & Base & 0.1 & No & 2 & $10^{8}$ & 0.2 & No & SSM \\
\hline S11 & High & 0.1 & Earlier & 0.1 & No & 2 & $10^{8}$ & 0.1 & No & SSM \\
\hline $\mathrm{S} 12$ & High & 0.1 & Later & 0.1 & No & 2 & $10^{8}$ & 0.1 & No & SSM \\
\hline $\mathrm{S} 13$ & High & 0.1 & Base & 0.1 & $t=4,5,6$ & 2 & $10^{8}$ & 0.1 & $t=4,5,6$ & SSM \\
\hline S14 & High & 0.1 & Base & 0.1 & $t=3,4,5,6$ & 2 & $10^{8}$ & 0.1 & $t=3,4,5,6$ & SSM \\
\hline S15 & High & 0.1 & Base & 0.1 & No & 2 & $10^{8}$ & 0.1 & No & OBS \\
\hline S16 & High & 0.1 & Base & 0.1 & No & 2 & $10^{8}$ & 0.1 & No & PROC \\
\hline
\end{tabular}

Cells with emboldened typeface indicate characteristics that changed in reference to scenario S1 (either in the simulation or the estimation phase). All scenarios used $q=10^{-7}$ to simulate abundance indices, and $\alpha_{R}=1 / 3$ to simulate $R$. CV is the coefficient of variation for both observation and process errors. We used the relationship $\sigma^{2}=\log \left(C V^{2}+1\right)$ that works for lognormal distributions. In $\mathrm{S} 1$, the exploitation rate is fixed at 0.3 month ${ }^{-1}$, and $M=$ 0.1 month $^{-1}$, generating a depletion rate of $50 \%$ after 3 months and $80 \%$ after 10 months. In $\mathrm{S} 2$, the exploitation rate is fixed at 0.13 month ${ }^{-1}$, generating a depletion rate of $30 \%$ after 3 months and $60 \%$ after 10 months. In all scenarios except S11 and S12, the parameter $\lambda$ used for the recruitment rhythm is the one given in the Methods section. Data for $S 11$ and $S 12$ were simulated by using a series $\left\{\lambda_{t}\right\}_{t=1, \ldots, n}$ that is 1 -month lagged backward and forward, respectively, from the reference rhythm. For estimation purposes, all priors are defined in Table 2, and all scenarios are fitted with the reference recruitment rhythm $\lambda$. S15 is fitted under the hypothesis of there being observation error only, and S16 of there being process stochasticity only.

$\theta$ given the data of the simulation $s$. For each scenario, $\operatorname{MRE}(\theta)$ is interpreted as the relative bias [Equation (4b)]:

$$
\begin{aligned}
\operatorname{RE}\left(\theta_{s}\right) & =\frac{E\left(\theta \mid \mathrm{data}_{s}\right)-\theta_{\text {true }}}{\theta_{\text {true }}} \text { for } s=1, \ldots, 50, \\
\operatorname{MRE}(\theta) & =\frac{1}{50} \sum_{s=1}^{50} \operatorname{RE}\left(\theta_{s}\right) .
\end{aligned}
$$

Second, the mean standard deviation (MSD) was calculated [Equations (5a) and (5b)], to measure the precision of the Bayesian estimation:

$$
\begin{aligned}
\operatorname{SD}\left(\theta_{s}\right) & =\sqrt{\operatorname{Var}\left(\theta \mid \mathrm{data}_{s}\right)} \text { for } s=1, \ldots, 50, \\
\operatorname{MSD}(\theta) & =\frac{1}{50} \sum_{s=1}^{50} \operatorname{SD}\left(\theta_{s}\right) .
\end{aligned}
$$

Third, for each scenario, we computed the standard deviation of the 50 posterior medians, denoted SDE, providing a measure of the variability of the estimates in the 50 simulations.

\section{The 16 scenarios}

In all, 16 contrasting scenarios (summarized in Table 3) were defined by adopting different specifications of parameters $M$, $\left\{\lambda_{t}\right\}_{t=1, \ldots, n},\left\{h_{t}\right\}_{t=1, \ldots, n}$, and $\sigma^{2}$ (simulation and estimation steps), and different specification of priors for $N_{1}, R, q$, and $\sigma^{2}$ (estimation step). For illustration, the 50 trajectories of abundance $\left\{N_{t}\right\}_{t=1, \ldots, n}$, catches $\left\{c_{t}\right\}_{t=1, \ldots, n}$, and abundance indices $\left\{h_{t}\right\}_{t=1, \ldots, n}$ obtained for three contrasting scenarios are plotted on Figure 3 . The value of hierarchical modelling has been demonstrated previously
(Rivot and Prévost, 2002; Gelman et al., 2004; McAllister et al., 2004), so the performance of hierarchical modelling was not explored through the SE approach. The value of the SSM approach in analysing sequential fishery data has also been demonstrated in other applications (see Punt, 2003, for a recent application to surplus production models). Therefore, SSM was our favoured default approach and the SE study mainly focused on contrasting scenarios designed to investigate five key issues:

(i) Are the estimates sensitive to the quantity of information contained in the dataset? We focused on two particular issues, the strength of depletion in the abundance index trajectory, and the level of process and observation noise.

(ii) Are the inferences sensitive to the prior on $N_{1}$ ?

(iii) Are the inferences sensitive to mis-specification of $M$ and of the secondary recruitment rhythm?

(iv) Are the inferences sensitive to the presence of missing data in the abundance index time-series as a result of fishing moratoria?

(v) Are the inferences sensitive to the error structure in the model? We compared the SSM estimation method with the observation error method (OBS) that ignores the process errors in the statistical procedure, and with the process error method (PROC) that ignores the observation errors.

Reference configuration (S1). All scenarios are variants of the reference configuration S1 that was built from expertise and considered to represent an archetype for the analysis of the Moroccan octopus fishery data. To simulate configuration S1, the exploitation rate was set to be high $\left(h=0.3\right.$ month $\left.^{-1}\right)$, leading to strong 
(a)
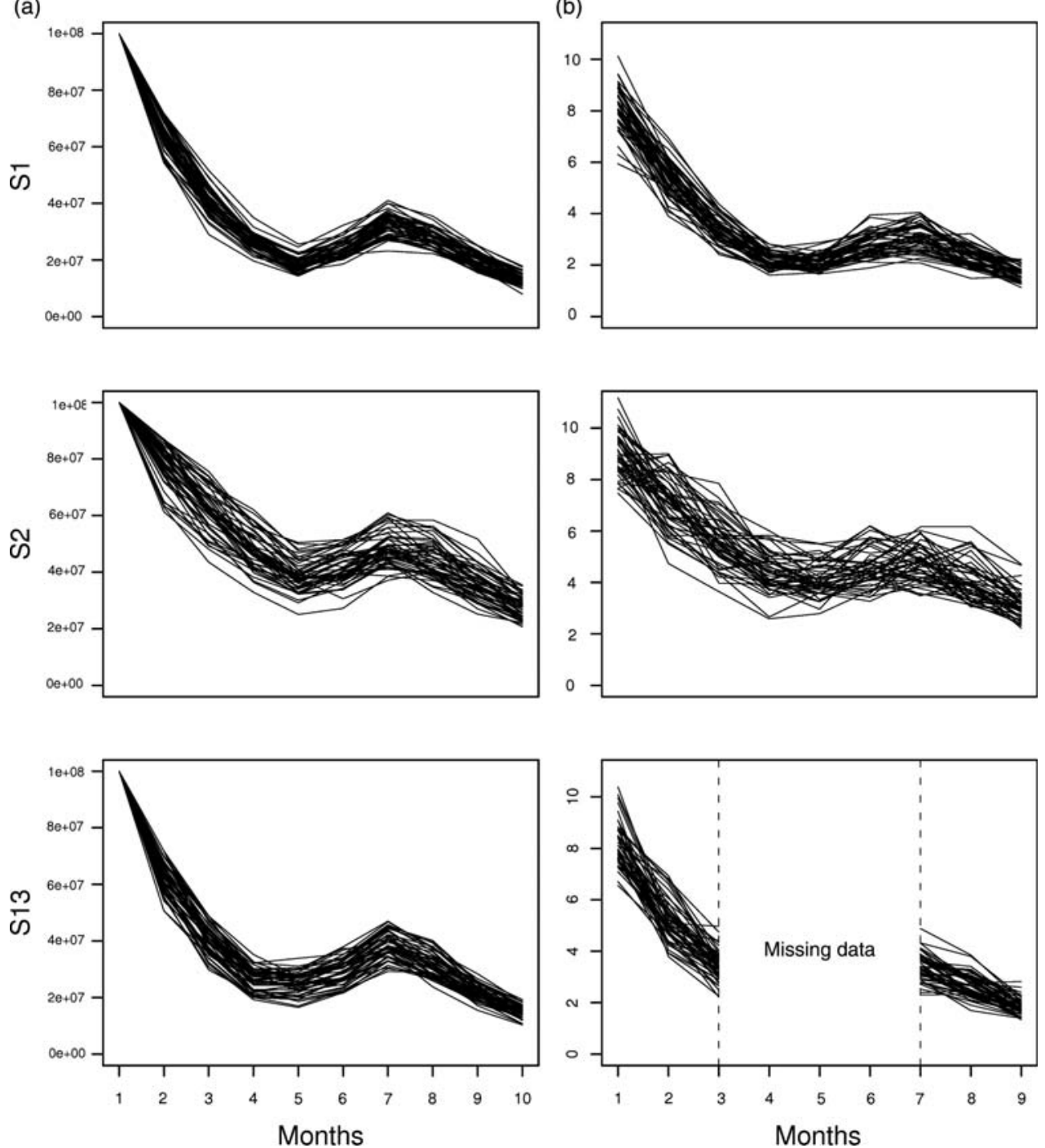

Figure 3. The SE approach, showing the shape of the 50 simulated trajectories for (a) abundance (in numbers), and (b) abundance indices (thousands per day at sea) for three representative scenarios S1, S2 (lower depletion), and S13 (fishing moratorium during months 4, 5, and 6).

depletion in the abundance index as a result of catch removals: 50 and $80 \%$ of the initial abundance was depleted after 3 and 10 months, respectively. $M$ was fixed at 0.1 , and $\left\{\lambda_{t}\right\}_{\mathrm{t}=1, \ldots, n}$ peaked in April (Figure 1). Process and observation noise were moderate, with a $10 \%$ coefficient of variation $\left(C V_{\mathrm{o}}=C V_{\mathrm{p}}=0.1\right)$. The model used for the estimation phase is the same as that used for the simulation (same values of $M$ ), $\left\{\lambda_{\mathrm{t}}\right\}_{\mathrm{t}=1, \ldots, n}$, and the ratio of the process and observation error variances was fixed at 1 . Catches and abundance indices were available for the 10 months, with no missing data. The prior distributions used were those defined in Table 2, and their mode (when it existed) corresponded to the value of the parameter used in the simulation.

Influence of the quantity of information in the dataset (S2-S5). We assessed the sensitivity of the inferences to two key factors that monitor the quantity of information in the data: the depletion rate $\left(i_{n} / i_{1}\right)$, and the noise intensity $\sigma^{2}$. To address the sensitivity to depletion rate, we compared scenario S1 with scenario S2, characterized by a lower exploitation rate fixed at $h=0.13$ month $^{-1}$, leading to a lower depletion rate (only $30 \%$ of the initial abundance depleted after 3 months, and $60 \%$ after 10 months). A higher rate of natural mortality would lead to a faster depletion rate, but not necessarily to a more informative data configuration, because the proportion of the depletion attributed to catches would be smaller. To address this point, scenario S3 was built with all characteristics equal to $\mathrm{S1}$, but with a higher natural mortality rate, $M=0.2$. Two additional scenarios, $\mathrm{S} 4$ and $\mathrm{S} 5$, with increasing noise intensity $(C V=0.20$ and 0.30 , respectively) were compared with S1.

Sensitivity to the prior on $N_{1}(S 6-S 8)$. We assessed the sensitivity of the inferences to the choice of the prior on $N_{1}$. Scenarios S6-S8 used the same dataset as $\mathrm{S} 1$, but inferences were derived with different priors on $N_{1}$. In S6, the prior for $N_{1}$ is unbiased (mode $=$ simulated value $\left.=10^{8}\right)$, but it is weaker $(C V=5)$ than in S1. In S7 and S8, the models of the priors are biased by factor of $1 / 10$ or 10 , respectively, from the simulated value.

Sensitivity to the mis-specification of $M$ (S9 and S10). $M$ cannot be estimated from these data, so must be set to a constant in the model. We assessed the sensitivity of setting $M$ to an incorrect value in $S 9$ and S10. In S9, simulations used $M=0.2$ (data from S3), whereas the estimation was made with $M=0.1$. In $\mathrm{S} 10$, the 
Table 4. Prior distributions for the hierarchical models on the values of $N_{1}$ and $q$ with or without autocorrelation.

\begin{tabular}{|c|c|c|}
\hline \multirow{2}{*}{$\begin{array}{l}\text { Parameter } \\
\text { Exchangeable }\end{array}$} & \multicolumn{2}{|c|}{ Distribution } \\
\hline & & \\
\hline$N_{1}$ & $\begin{array}{l}\text { For } y=1, \ldots, n \\
\log \left(N_{1}[y]\right) \sim \text { normal }\left(\mu_{\log N}, \sigma_{\log N}^{2}\right)\end{array}$ & $\begin{array}{l}\mu_{\log N} \sim \operatorname{normal}(20, V=100) \\
\sigma_{\log N} \sim \text { uniform }(0,10)\end{array}$ \\
\hline 9 & $\begin{array}{l}\text { For } y=1, \ldots, n \\
\log (q[y]) \sim \text { normal }\left(\mu_{\log q}, \sigma_{\log q}^{2}\right)\end{array}$ & $\begin{array}{l}\mu_{\log q} \sim \operatorname{normal}(-11.5, V=100) \\
\sigma_{\log q} \sim \text { uniform }(0,10)\end{array}$ \\
\hline \multicolumn{3}{|c|}{ With random walk } \\
\hline$N_{1}$ & $\begin{array}{l}\text { For } y=1, \ldots, n \\
\log \left(N_{1}[y]\right) \sim \text { normal }\left(\mu_{\log N[y]}, \sigma_{\log N}^{2}\right) \text { with } \\
\mu_{\log N[y+1]} \sim \text { normal }\left(\mu_{\log N[y]}, \sigma_{\log N}^{2}\right)\end{array}$ & $\begin{array}{l}\sigma_{\log N} \sim \text { uniform }(0,10) \\
\mu_{\log N[1]} \sim \text { normal }(20, V=100) \\
\sigma_{\mu \log N} \sim \text { uniform }(0,10)\end{array}$ \\
\hline 9 & $\begin{array}{l}\text { For } y=1, \ldots, N \\
\log (q[y]) \sim \text { normal }\left(\mu_{\log q[y]}, \sigma_{\log q}^{2}\right) \text { with } \\
\mu_{\log q[y+1]} \sim \text { normal }\left(\mu_{\log q[y]}, \sigma_{\mu \log q}^{2}\right)\end{array}$ & $\begin{array}{l}\sigma_{\log q} \sim \text { uniform }(0,10) \\
\mu_{\log q[1]} \sim \text { normal }(-11.5, V=100) \\
\sigma_{\mu \log q} \sim \text { uniform }(0,10) \\
\end{array}$ \\
\hline
\end{tabular}

same simulated data as in S1 were used $(M=0.1)$, but the estimations were made with $M=0.2$.

Sensitivity to the rhythm of the secondary recruitment pulse (S11 and S12). The value $\left\{\lambda_{t}\right\}_{t=1, \ldots, n}$ cannot be estimated from the abundance index and catch data only, so must be fixed. However, as spawning season and growth rate of octopus vary year on year, the rhythm with which the secondary recruitment pulse arrives in the fishery is variable and uncertain. We assessed the sensitivity of the estimation method to mis-specification of $\lambda$. The data were simulated by using a series $\left\{\lambda_{t}\right\}_{t=1, \ldots, n}$ which is lagged 1 month earlier (S11) and 1 month later (S12) compared with the reference rhythm used for estimation.

Impact of missing data in the series of abundance indices (S13 and S14). In the Moroccan fishery, a fishing moratorium is set up during the breeding period. The measure aims to ensure a satisfactory level of recruitment for the following year. Months with $c_{t}=0$ and $i_{t}=\mathrm{NA}$ (not available) indicate that a fishing moratorium has been put in place. Scenarios S13 and S14 provide an assessment of the sensitivity of the inferences to the duration of the fishing moratorium. In S13, the moratorium is for months 4-6 (capturing situations observed in 2000), and in S14 in months 3-6 (capturing situations observed in 2001).

Sensitivity to the error structure (SSM, OBS, and PROC) (S15 and S16). Both S15 and S16 are based on the simulated data of S1 (operational model with both process noise and observation error). S1, S15, and S16 only differ in the error structure used during the fitting process. S15 corresponds to the OBS, where the model is fitted under the hypothesis of no process error ( $\sigma_{\mathrm{p}}^{2}=0$, observation error only), and S16 to the PROC, where the model is fitted under the hypothesis of no observation error $\left(\sigma_{\mathrm{o}}^{2}=0\right.$, process noise only).

\section{Analysis of the 21 Moroccan depletion time-series}

Five models of increasing complexity were applied to the Moroccan data, described in the following subsections.

\section{All depletion time-series treated as independent}

In the baseline model configuration, $M_{1}$, the 21 depletion timeseries were treated independently with the model described in Equations (1) and (2), the fixed parameters $M$ and $\lambda$ used for the reference scenario $S 1$ and the prior in Table 2.

\section{Hierarchical structure}

Based on the baseline model configuration $M_{1}$, four alternative hierarchical structures were designed, denoted as $M_{2}, \ldots, M_{5}$, in which the 21 depletion time-series were modelled jointly. In configuration $M_{2}$, we followed McAllister et al. (2004), and both the initial abundance for the 21 depletion series, the values of $N_{1}$ (with $y=1, \ldots, 21$ ) and catchability $q$ were modelled in an exchangeable hierarchical structure (Table 4).

In $M_{3}-M_{5}$, the hypothesis was introduced that the values of $N_{1}$ and $q$ form an autocorrelated RW. The fishing efficiency is not likely to show abrupt changes with time, but rather to show fluctuations caused by gradual changes in behaviour and development of the fishing fleet. Therefore, the values of $q$ were modelled as being drawn in a lognormal, with means (in log-scale) modelled as a normal RW (Table 4). Likewise, the values of $N_{1}$ are likely to show autocorrelated fluctuations because of the population dynamics and environmental influences. Hence, the values of $N_{1}$ were also modelled as lognormal with means (in log-scale) modelled as a normal RW (Table 4). Note that the variance parameter that underlies the RW is unknown, with a relatively uninformative prior on it. Hence, such models can fit abrupt changes in the series. In configuration $M_{3}$, RW is introduced only on the values of $q$, the values of $N_{1}$ being modelled as hierarchically exchangeable (Table 4). In $M_{4}$, RW is introduced on the values of $N_{1}$ only, the values of $q$ being modelled as exchangeable. In $M_{5}$, an RW was introduced on values of both $N_{1}$ and $q$.

To provide a sensitivity analysis of the inferences to misspecification of $M$ and $\lambda$, the estimation procedure was also run with $M$ set at 0.2 month $^{-1}$, and by considering a vector $\lambda$ lagged 1 month back (i.e. earlier) and 1 month forward (later) compared with the baseline rhythm.

\section{MCMC simulations}

All computations were performed with the R platform (www.Rproject.org). Given a series of catches $\left\{c_{t}\right\}_{t=1, \ldots, n}$ and abundance indices $\left\{i_{t}\right\}_{t=1, \ldots, n}$, the joint posterior distribution of all unknowns $p\left(N_{t=1, \ldots, n}, R, q, \sigma^{2} \mid\right.$ data $)$ can be approximated via the MCMC simulation method. Here, the mechanisms of the MCMC simulations are not detailed. Instead, we refer the reader to Meyer and Millar (1999) and other applications (e.g. Rivot et al., 2004; Hammond and Trenkel, 2005; Swain et al., 2009), who showed how the Metropolis-Hastings within Gibbs 
(a)

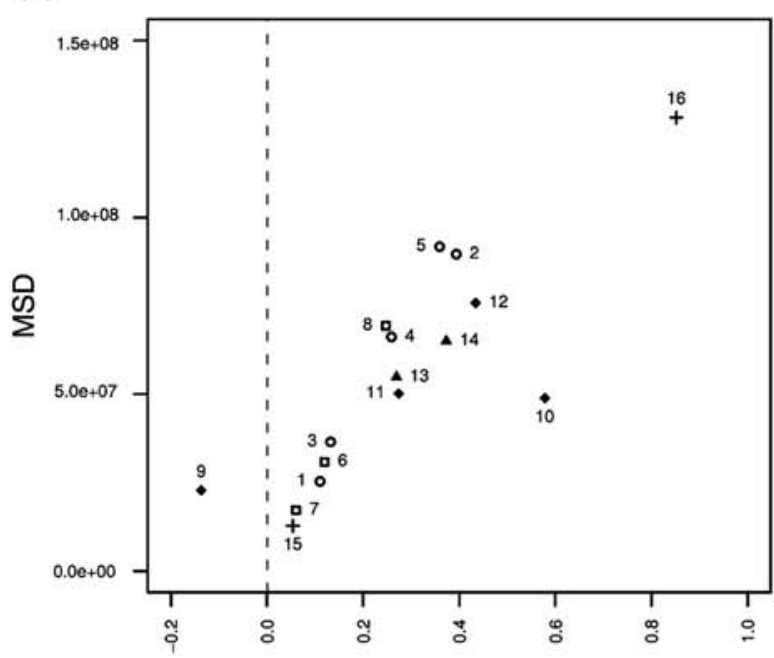

(d)

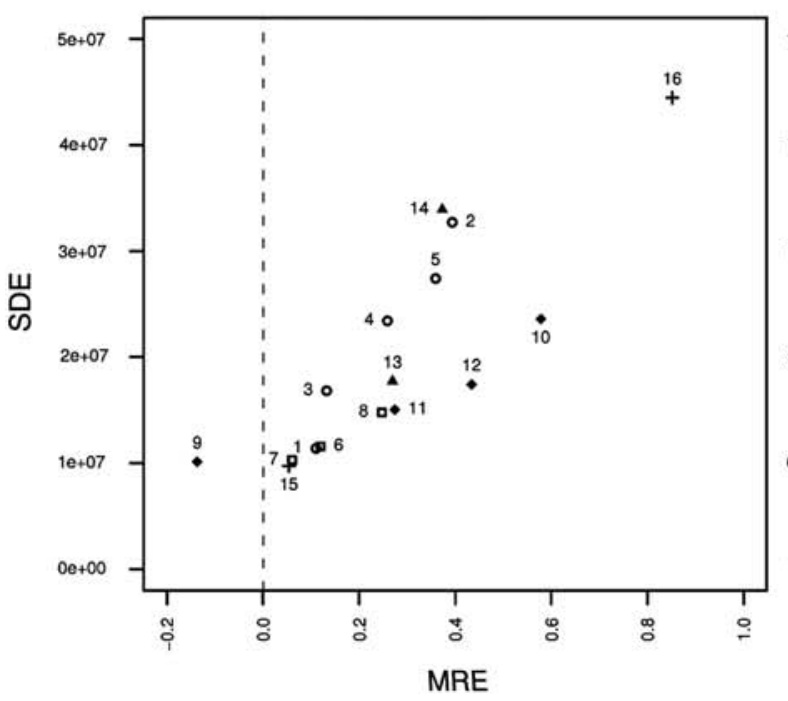

(b)

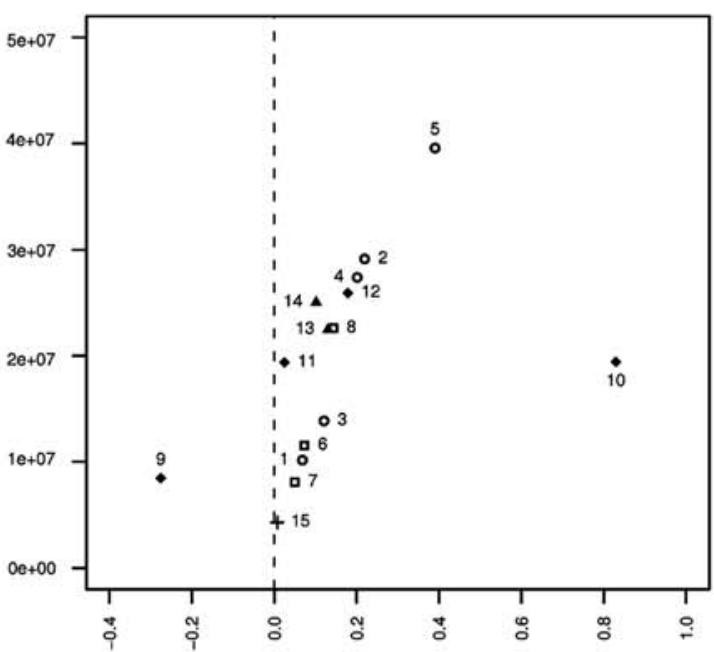

(e)

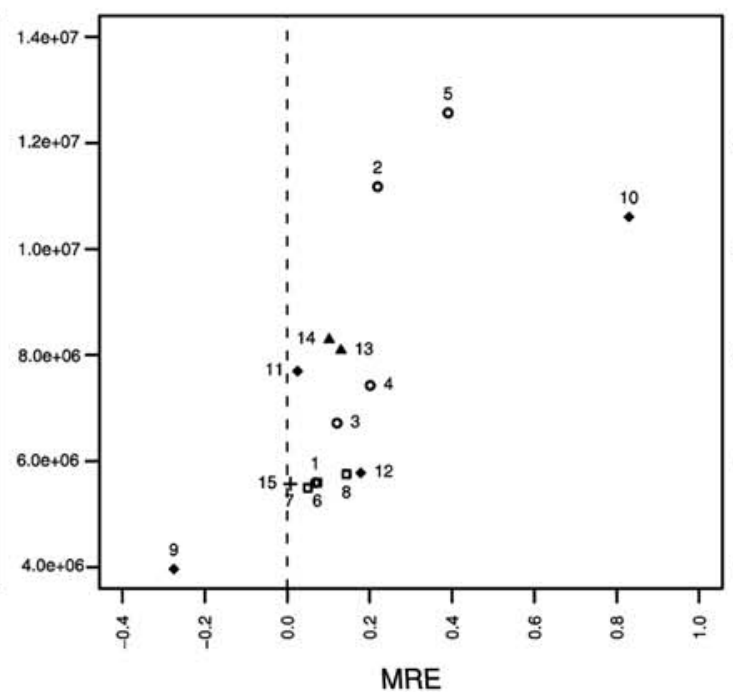

(c)

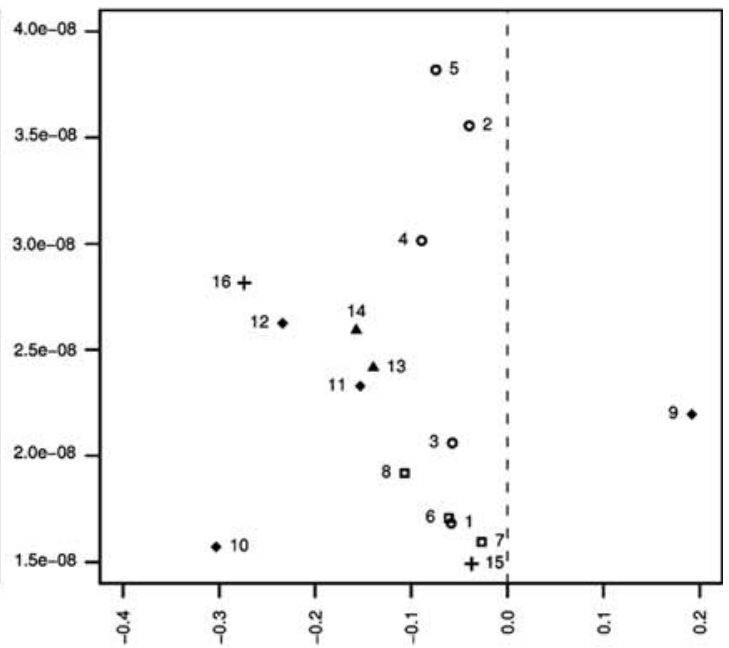

(f)

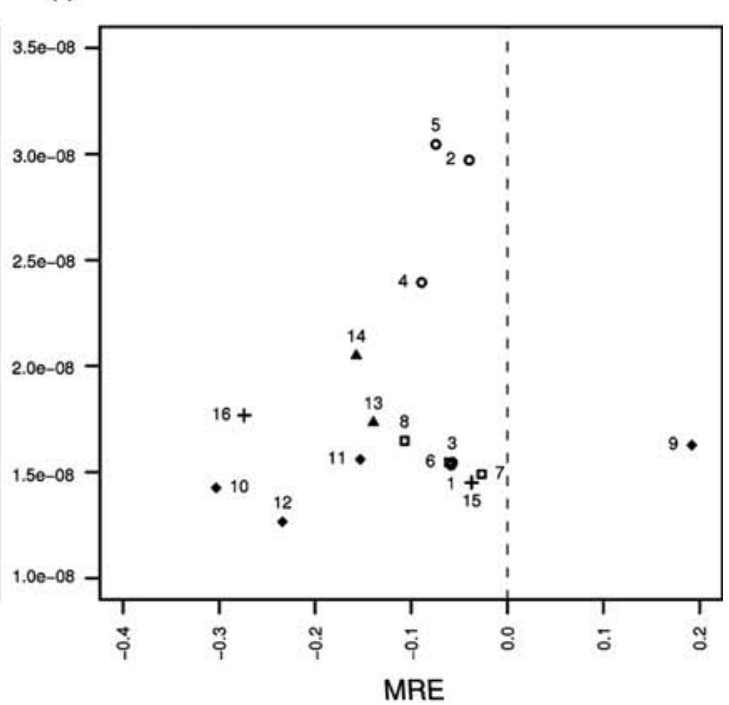

Figure 4. The SE approach, showing the performance of the estimation method measured on (a and $\mathrm{d}) N_{1}$, ( $\mathrm{b}$ and $\mathrm{e}$ ) $R$, and (c and $\left.\mathrm{f}\right) q$, for the 16 scenarios tested. The performance is measured by MRE ( $x$-axis) and MSD ( $y$-axis, top panels), and SDE ( $y$-axis, bottom panels). Scenarios are plotted using symbols related to the four issues they address. Open circles, influence of the quality of information in the dataset $(\mathrm{S} 1, \ldots, \mathrm{S} 5)$; open squares, sensitivity to the prior on $N_{1}(\mathrm{~S} 6, \ldots, \mathrm{S} 8)$; diamonds, sensitivity to mis-specification of $M$ and $\lambda_{t}$ (S9, ..., S12); triangles, sensitivity to the duration of the fishing moratorium (S13 and S14); crosses, observation error and process error methods (S15 and S16; the MRE, MSD, and SDE criteria for R in S16 are not shown because they are outside, i.e. highly positive values, the range of the graph). 

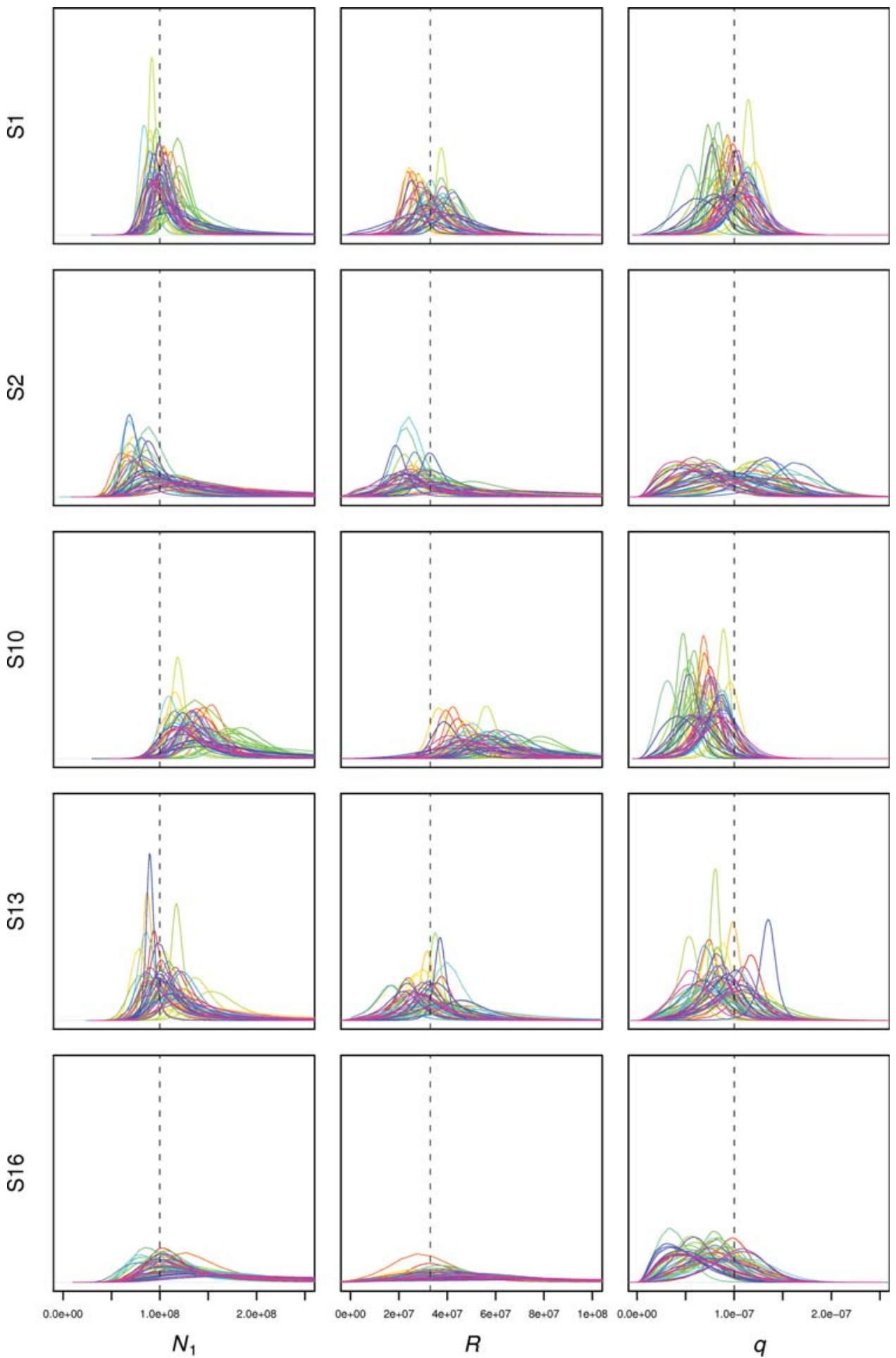

Figure 5. The SE approach, showing profiles of the marginal posterior probability distributions of key parameters $N_{1}, R$, and $q$ for scenarios $S 1$, S2 (lower depletion), S10 (mis-specification of $M=0.2$ ), S13 (fishing moratorium during months 4, 5, and 6), and S16 (estimation under the process error model assumption). For each scenario and each parameter, 50 posterior distributions corresponding to 50 simulation trials are plotted. The vertical line represents the true values used in the simulations.

algorithm implemented in BUGS software family (Thomas et al., 1992; Lunn et al., 2009) can be used to fit non-linear non-Gaussian SSMs. All the Bayesian estimations (both the SE approach and the application to Moroccan data) were derived using the OpenBUGS 3.0.3 software (available at http://mathstat .helsinki.fi/openbugs/) through the BRugs package, which allowed us to run OpenBUGS from the R platform.

For all estimations, three MCMC-independent chains with dispersed initialization points were used. For each chain, the first 100000 iterations were discarded. After this "burn-in" period, 
only one in 10 iterations was kept to reduce the MCMC sampling autocorrelation. Inferences were then derived from a sample of 30000 iterations derived from three chains of 10000 iterations. All the modelling results have undergone tests to assess convergence of MCMC chains (the Coda package of R). It is therefore assumed that the reported posterior probability density functions are representative of the underlying stationary distributions.

The calculation for the SE approach was time-consuming; each scenario (50 estimations procedure) took $\sim 6 \mathrm{~h}$ (Processor Intel Duo-core $2 \mathrm{Gz})$.

\section{Results \\ SE approach}

The MRE, MSD, and SDE values obtained for the three key parameters $N_{1}, R$, and $q$ and for the 16 scenarios tested are summarized in Figure 4. The marginal posterior distributions of $N_{1}$, $R$, and $q$ obtained for five contrasting scenarios are shown in Figure 5 .

\section{Inferences are sensitive to the quantity of information in the data}

Results showed that the strength of the depletion at the start of the time-series critically determines the performance of the estimation method. Inferences are generally unbiased, precise, and reliable when the data show a strong depletion in response to catch removals. When depletion becomes weaker, identifiability problems appear between $q$ and $N_{1}$, and the estimation may fail. Estimates of $q$ are likely to be negatively biased, corresponding to positively biased estimates of $N_{1}$ and $R$. Comparison between the inferences obtained in S1 and S2 illustrates this general conclusion (Figure 4). Compared with S1, which provides the most information for parameter estimation, S2 leads to a 40 and $20 \%$ overestimate of $N_{1}$ and $R$, respectively. The inferences on $q$ are also of poor quality. For the three parameters, the variance of the estimates is much higher for S2 than for S1, indicating that the inferences are less reliable (Figure 4). Comparison between S1 and S3 (Figure 4) shows that the data in S3 become less informative and the performance of the depletion method declines because more of the depletion is attributable to natural mortality and less to fishing. Estimation performance was sensitive to the intensity of noise in the data. Comparison between S1, S4, and S5 (Figures 4 and 5) illustrates how the quality of the inferences deteriorates with increasing noise intensity, in terms of biases, precision, and reliability.

\section{Inferences are not sensitive to prior speciation on $N_{1}$}

For a rather informative dataset characterized by a strong depletion rate, inferences are robust to the prior on $N_{1}$ (e.g. S1 vs. S6, S7, and S8). Even prior distributions with biased modes (S7 and S8) led to relatively unbiased and precise posteriors.

Sensitivity to mis-specification of $M$ and $\lambda$ is moderate to high The performance of the estimation method can be reduced if $M$ (natural mortality) and $\lambda$ (the rhythm of the secondary recruitment) are mis-specified. Inferences appear to be highly sensitive to mis-specification of $M$. Results from S9 and S10 demonstrated that overestimating $M$ in the estimation procedure (S10) resulted in positively biased estimates of $N_{1}$ $(60 \%)$ and $R(80 \%)$, and in negatively biased estimates of $q$ (Figure 5). To a lesser extent, underestimating $M$ (S9) results in underestimates of $N_{1}$ and $R$. The sensitivity to overestimating
$M$ is because a larger value of $M$ decreases the fraction of the depletion attributed to catch removal, so reduces the information in the data.

Mis-specification of $\lambda$ seems to have less of an impact on the performance of the estimation (S1 vs. S11 and S12). Both $R$ and $N_{1}$ are overestimated when $\lambda$ is mis-specified, such that it is thought to occur too early (S11) or too late (S12), but the bias in $R$ is always $<20 \%$.

\section{Inferences remain accurate even with missing data}

Comparing scenarios S1 with S13 and S14 shows that the performance of the estimation drops when the proportion of missing data increases. Yet, results suggest that if the depletion rate in the series of abundance indices is high, the performance remains relatively good. Reliable inferences are obtained even if the moratorium begins early in the fishing season (S14).

\section{Performance of the three estimation methods,} SSM, OBS, and PROC

The results demonstrate the very poor estimation performance of the PROC (S16). The bias is positive for $N_{1}$ and $R$, and negative for $q$, and is large for all three parameter estimates (Figure $4 \mathrm{~b}$ and $\mathrm{d}$ ). The MRE, MSD, and SDE criteria for $R$ in S16 are not shown because they are outside the range of the graph. The posterior distributions of $N_{1}, R$, and $q$ are widely dispersed (Figure 5), and the variance of the point estimates between the 50 simulations is also high. In contrast, the three criteria MRE, MSD, and SDE do not show clearly that the SSM method outperforms the OBS (S15). However, the MSD criterion (slightly lower for S15) combined with a precise examination of the shape of the posterior distributions (not shown) indicate that the OBS generally produces tighter (though not less biased) posterior distributions than the SSM method. In other words, the OBS produces more "incorrectly precise" distributions than the SSM method, so increasing the risk of deriving biased inferences. The SSM method produces more dispersed and slightly less biased posterior distributions, so appears to be a more robust inference approach in a context of multiple sources of uncertainty.

\section{Conclusions}

The performance of the BSSM estimation method was satisfactory under most conditions considered. In nearly all scenarios, biases on $R$ rarely exceeded $40 \%$. Inferences on $N_{1}$ and $q$ were slightly more sensitive than those on $R$, with relative biases of $N_{1}$ that rarely exceeded $50 \%$. Our results corroborate identifiability problems between $q$ and $N_{1}$ (Gonzalez Cano and Restrepo, 2001; McAllister et al., 2004; Magnusson and Hilborn, 2007) for noninformative data. Low depletion rates and/or high noise intensity lead to negatively biased and imprecise estimates of $q$, which in turn lead to positively biased estimates of $N_{1}$.

The octopus is intensely exploited in Moroccan waters, producing strong depletion rates during the fishing season (depletion rate is $>70 \%$ for most fishing seasons; see Figure 2). Given the results of the SE analysis, the strong depletion in the Moroccan data may guarantee a good fit of the model for most of the 21 depletion series. In the second part of the series, fishing moratoria become more frequent. In particular, in the final three depletion series, only two time-steps are available, before a 3- or a 4-month closure. However, the results of the SE approach revealed that reliable inferences can be obtained even in such apparently data-poor situations. 
(a)
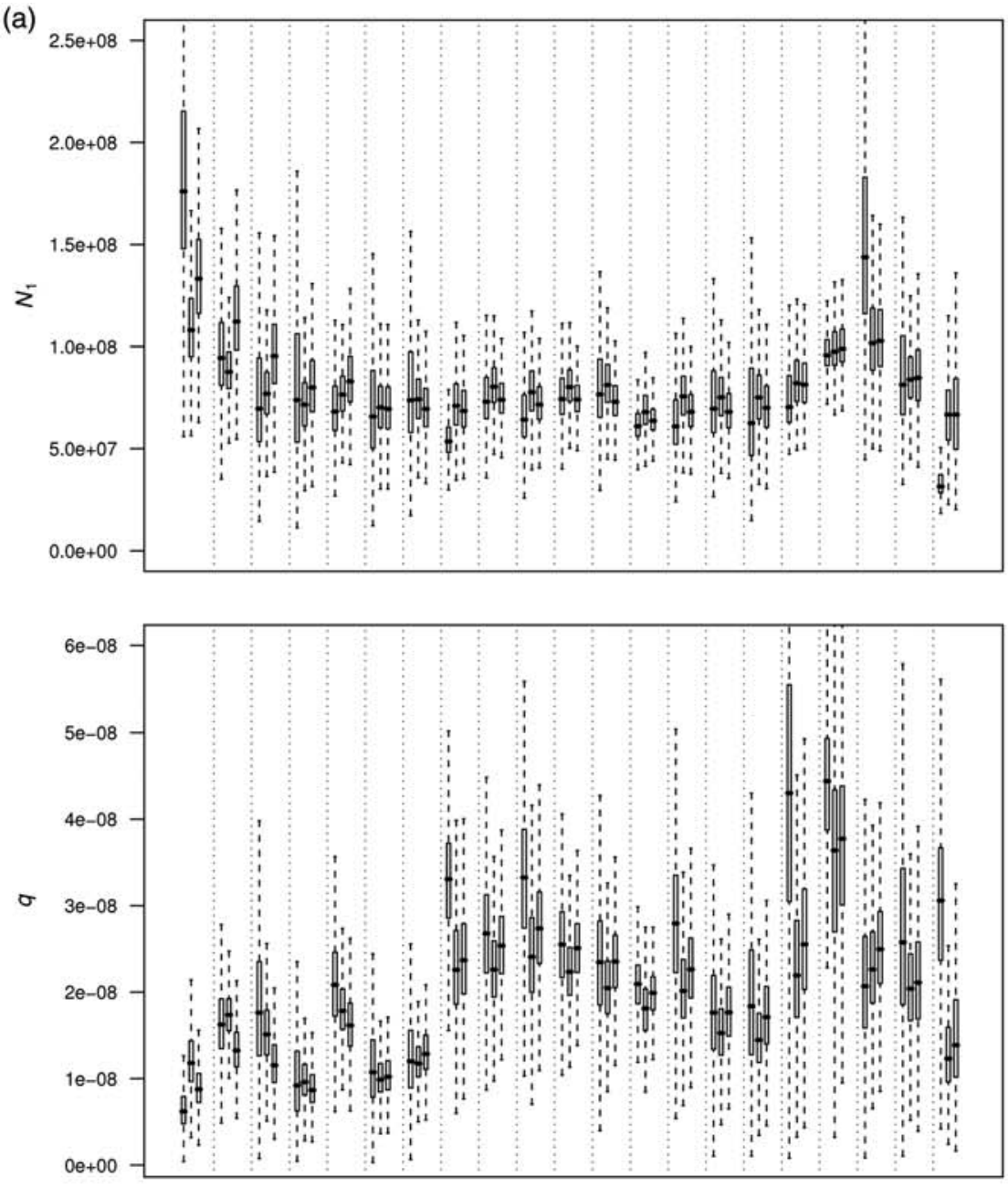

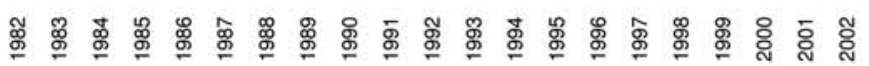
Depletion series (b)
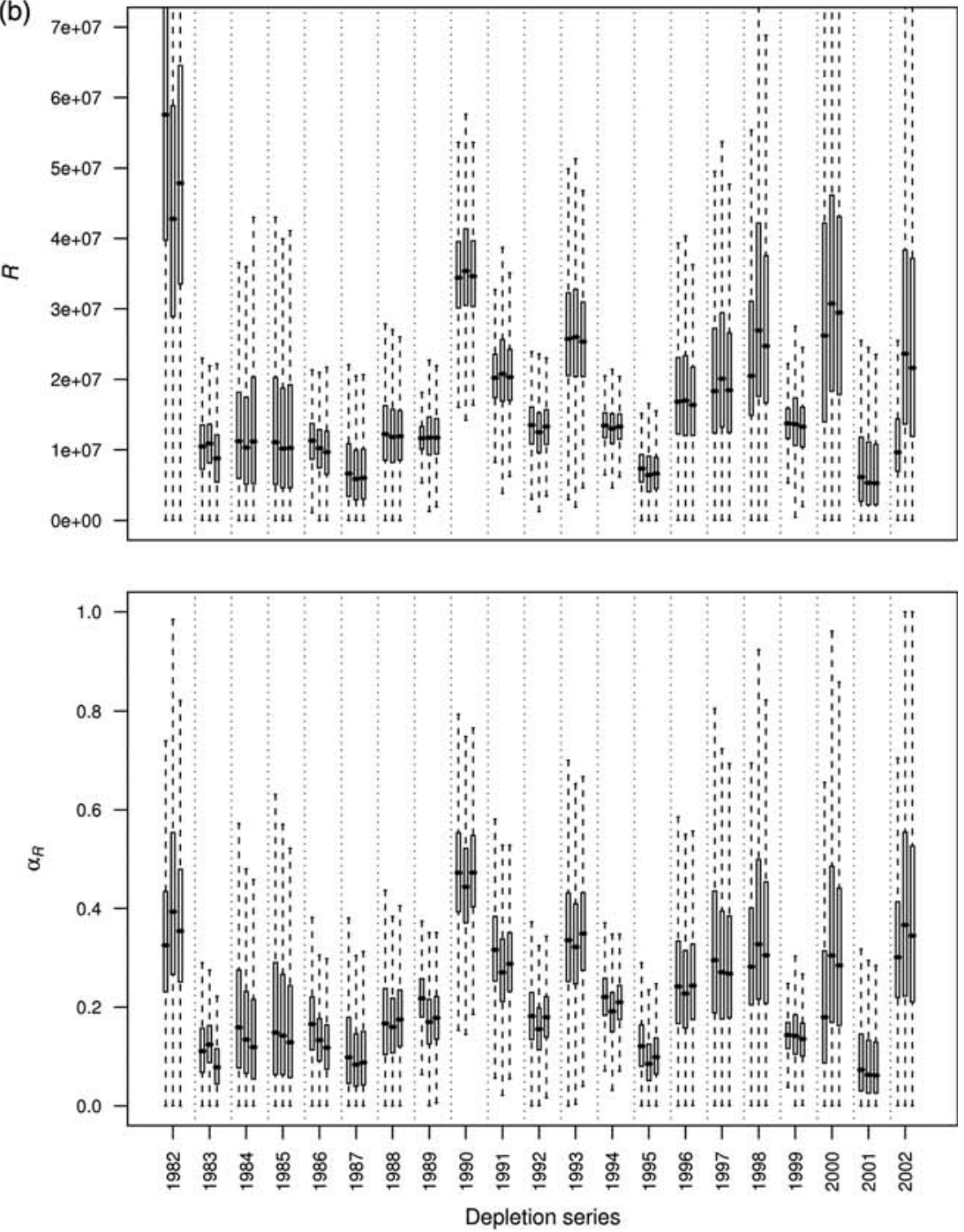

Figure 6. Analysis of the Moroccan dataset, showing the sensitivity of the inferences on (a) $N_{1}$ and $q$, and (b) $R$ and $\alpha_{R}$ to the model configurations $M_{1}, M_{2}$, and $M_{5}$. For each of the 21 depletion series $\left(x\right.$-axis), the three box-plots represent the posterior distributions obtained under the alternative model configurations in the following order: $M_{1}$, all depletion series are independent; $M_{2}$, exchangeable hierarchical structure on the values of $N_{1}$ and $q ; M_{5}$, RW on the values of $N_{1}$ and $q$. 


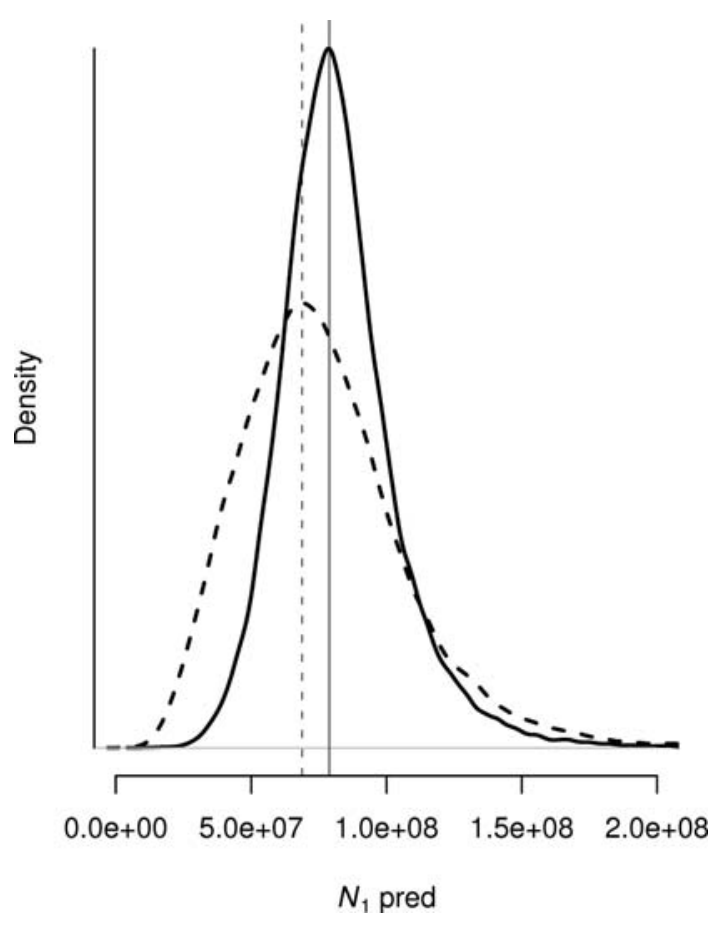

Figure 7. Analysis of the Moroccan dataset, showing posterior predictive distributions of the initial abundance $N_{1}$ derived from the exchangeable hierarchical model $M_{2}$ (solid line) and from the hierarchical model with RW $M_{5}$ (dotted line).

The SE findings also flag some potential problems that may arise in the analysis of the Moroccan dataset. For some depletion series (e.g. 1982-1983, 1985-1986), the depletion rate is low and one must interpret the inferences with caution. The results also show that the inferences on $R$ and $N_{1}$ might be sensitive to mis-specification of $M$ and the rhythm of the secondary recruitment $\lambda$. Therefore, our estimation method using fixed values for $M$ and $\lambda$ needs to be accompanied by a sensitivity analysis to these parameters. Note too that the performance of the estimation method deteriorates when noise in the process and the data increases. As no information on the amount of noise associated with the dataset is available, results have to be interpreted with caution.

\section{Applications to the Moroccan dataset}

\section{Model $M_{1}$ : all depletion series treated independently}

For almost all depletion series, model configuration $M_{1}$ provides sensible inferences for key parameters. The uncertainty about the estimates is low except for the depletion series 1982 and 2000 (Figure 6a). Note, though, that the model provides quite precise estimates of $N_{1}$ even for the last 2 years characterized by many missing data as a result of a long fishery closure. The medians of the posterior estimates of initial abundance $N_{1}$ vary among years around an average of $\sim 80$ million animals (Figure $6 a$ ), with autocorrelated variations in the time-series.

Marginal posterior distributions of $q$ (Figure 6a) suggest great variability of this parameter among years. However, this consideration should be cognizant of the high negative correlation in the joint posterior distributions of $\left(N_{1}, q\right)$.

The model also provides sensible estimates of the secondary recruitment $R$. The values of $\alpha_{R}$ are well estimated. On average, the secondary recruitment is $22 \%$ of the principal recruitment (Figure 6b). The median of the posterior estimates of $R$ varies among years around an average of $\sim 17$ million animals (Figure $6 \mathrm{~b}$ ). The uncertainty about $R$ is higher than the uncertainty about $N_{1}$, in particular for the depletion series 1982, 1998, 2000, and 2002. The joint posterior distributions of $N_{1}$ and $R$ do not reveal strong correlation patterns (not shown).

The estimates of the $C V$ vary around 0.20 (not shown). According to the results from the SE approach, such noise intensity in the process and observations should allow one to remain confident in the quality of the inferences.

Hierarchical models $M_{2}, \ldots, M_{5}$

Four alternative hierarchical structures were tested in the model configurations $M_{2}, M_{3}, M_{4}$, and $M_{5}\left(M_{3}\right.$ and $M_{4}$ are not shown). In model $M_{2}$, values of both $N_{1}$ and $q$ were modelled in an exchangeable hierarchical structure. As expected from a hierarchical model (Rivot and Prévost, 2002; McAllister et al., 2004), the estimates of $N_{1}$ and $q$ dropped towards the overall mean compared with the estimates obtained from the configuration $M_{1}$ (Figure 6a). The shrinkage effect is higher for years with less informative depletion series (e.g. 1982 and 2000), the hierarchical model $M_{2}$ providing much less uncertain posterior inferences than $M_{1}$ on $N_{1}$. Setting a hierarchical structure on the values of $N_{1}$ and $q$ has relatively little effect on the estimates of $R$ and $\alpha_{R}$ (Figure 6b).

The posterior prediction for $N_{1}$ obtained from hierarchical model $M_{2}$ (Figure 7) reflects the amount of information provided by the data from all depletion series. It can be used as a prior to predict the initial abundance for an unsampled year. The mode is $\sim 0.75 \times 10^{8}$, with $50 \%$ of the probability between $0.68 \times 10^{8}$ and $0.93 \times 10^{8}$.

The key hypothesis behind the exchangeable hierarchical structure is that conditional on the hyperparameters, the values of $N_{1}$ are iid. However, the time-series of $N_{1}$ and $q$ obtained from the exchangeable hierarchical model $M_{2}$ reveals fluctuations with positive lag- 1 autocorrelation coefficients $\left(0.15\right.$ and 0.20 for $N_{1}$ and $q$, respectively). As expected from this observation, models $M_{3}, M_{4}$, and $M_{5}$, which introduce RW in the time-series of the $N_{1}$ and $q$ was preferred to the exchangeable hierarchical model $M_{2}$. Indeed, the deviance information criterion (Spiegelhalter et al., 2002; Rivot et al., 2008) computed for the four model configurations are, respectively, $-311,-313,-315$, and -318 , indicating that $M_{5}$ can be expected to predict the data most accurately among the models tested.

The posterior estimates obtained from $M_{5}$ do not drastically differ from those of $M_{2}$ (Figure $6 \mathrm{a}$ and $\mathrm{b}$ ). The posterior predictive distribution for $N_{1}$ obtained from $M_{5}$ (Figure 7) gives maximum credibility to slightly lower values of $N_{1}$. This is easily explained because of the lag-1 autocorrelation: the predicted $N_{1}$ for 2003 (the next but yet unsampled year) depends on the value of $N_{1}$ from the previous year (2002), which was slightly lower than the overall mean.

\section{Total abundance and exploitation rates}

The model provides abundance estimates for each month since 1982 (Figure $8 \mathrm{a}$ ). The effect of secondary recruitment is visible on the time-series of estimated abundance within each season (see for instance 1990 and 1996). The time-series of point estimates of the principal and secondary recruitment pulses exhibit smooth fluctuations (Figure $8 \mathrm{~b}$ ). 
(a)

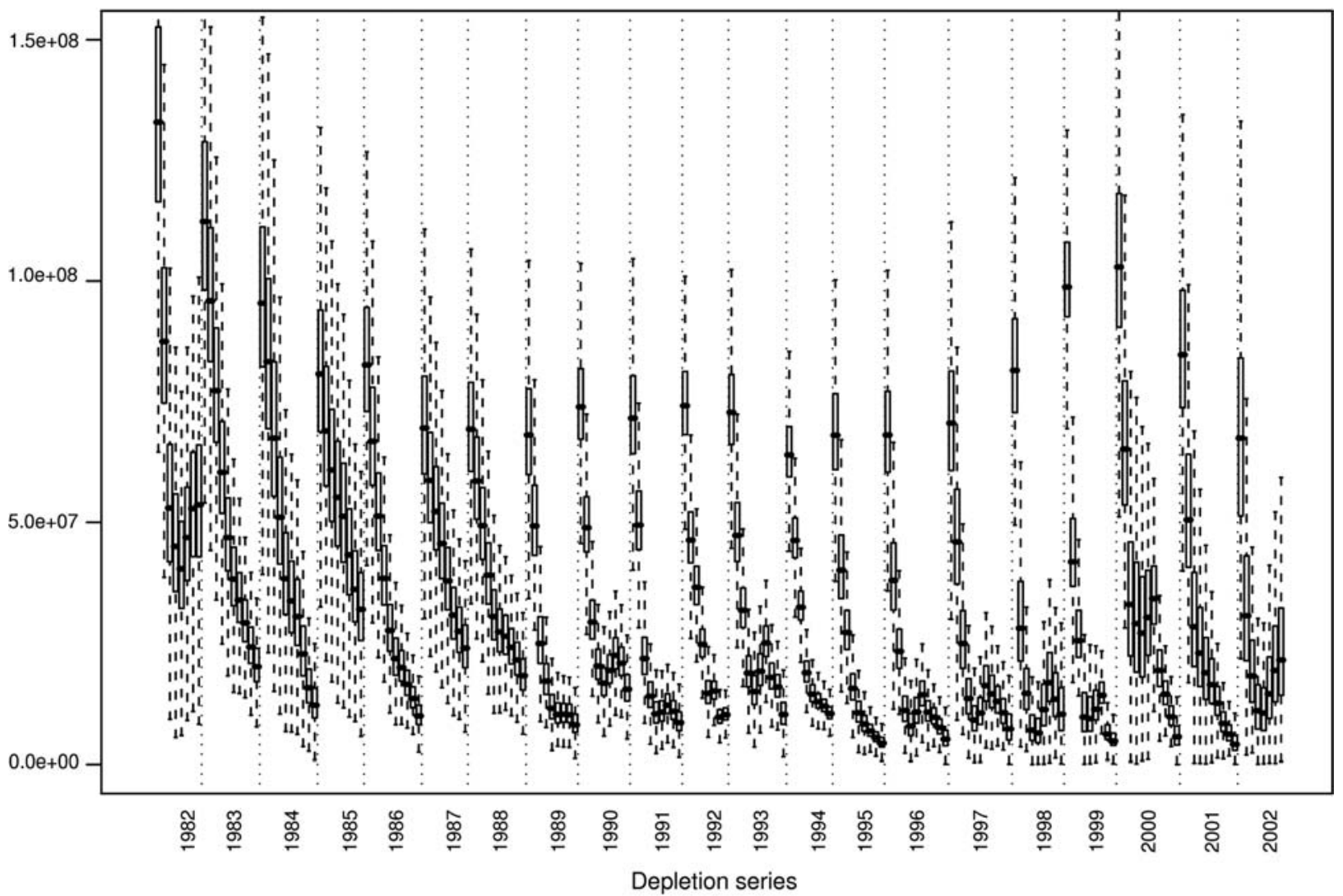

(b)

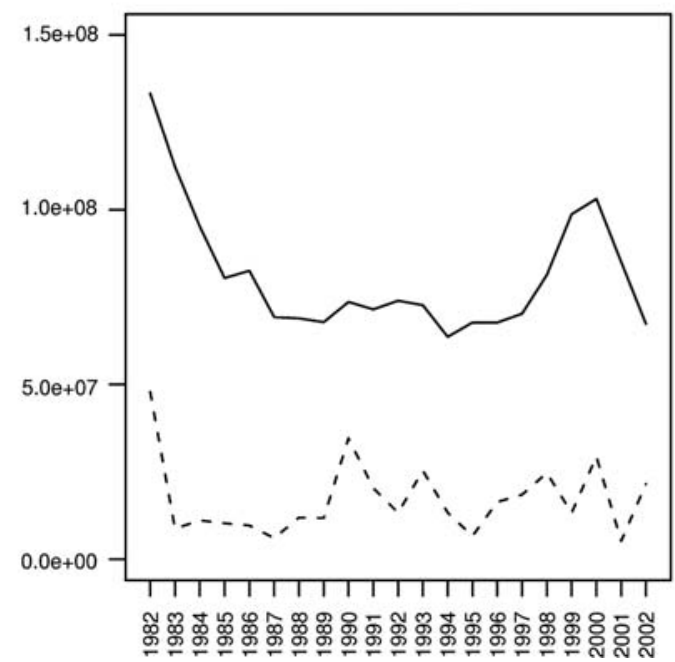

(c)

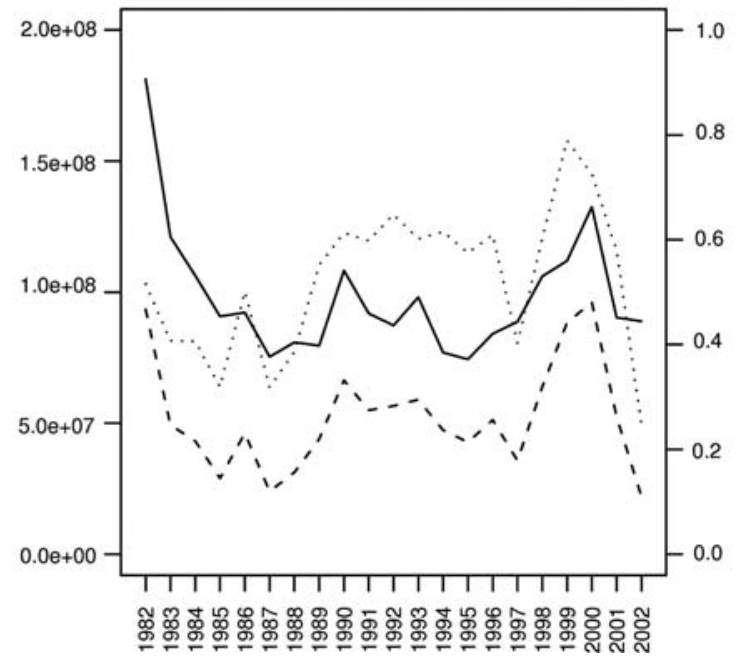

Figure 8. Analysis of the Moroccan dataset, showing the time-series ( 21 depletion series) of point estimates of $N_{1}, R$, and a comparison of the total recruitment with annual catches. (a) Box-plot of the posterior distributions of the number of octopus taken each month between 1982 and 2002; (b) Posterior medians of $N_{1}$ (solid line) and $R$ (dashed line) by number; (c) Left axis (solid line), posterior medians of total recruitment $N_{1}+R$ and total annual catches (dashed line) by number; right axis (dotted line), harvest rate estimated as the ratio of the annual catches vs. the posterior median of the total recruitment $\left(N_{1}+R\right)$.

Comparing the annual catches with estimates of total recruitment $\left(N_{1}+R\right)$ allows us to estimate the exploitation rate. Estimated annual exploitation rates range from 25 to $80 \%$ (Figure 8c), consistent with earlier observations suggesting intense exploitation of octopus in Morocco (Faraj et al., 2006). The exploitation rates exhibit a slightly increasing trend over the time-series, consistent with the expertise available to the fishery. 

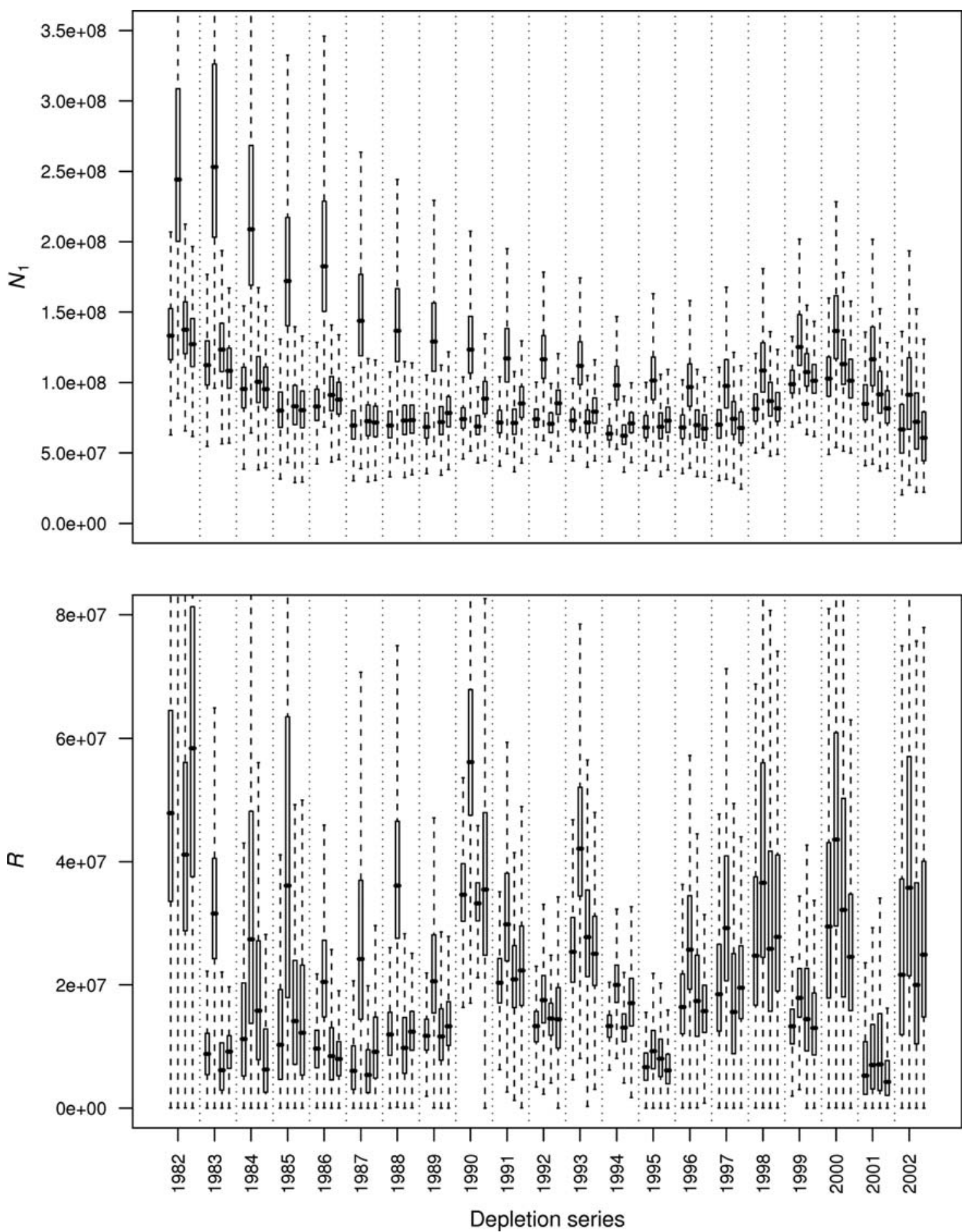

Figure 9. Analysis of the Moroccan dataset, showing the sensitivity of the inferences on $N_{1}$ and $R$ to $M$ and $\lambda$. For each of the 21 depletion series ( $x$-axis), the four box-plots represent the posterior distributions obtained under four model configurations in the following order. First, $M_{5}$, RW on the values of $N_{1}$ and $q$, with $M=0.1$ and baseline recruitment rhythm $\lambda$; second, $M_{5}$ with $M=0.2$; third, $M_{5}$ with $\lambda$ 1-month lagged earlier by reference to the reference rhythm; fourth, $M_{5}$ with $\lambda$ lagged later by 1 month.

Sensitivity analysis in $M_{5}$

Conclusions relative to the sensitivity to $M$ and $\lambda$ are qualitatively the same as in the SE approach (Figure 9). The main caveat for the model is that inferences look very sensitive to the values of $M$. In contrast, for almost all years, the results look rather robust to changes in $\lambda$.
The sensitivity of the results to the prior on the key parameters $N_{1}$ and $q$ was also checked by running model $M_{5}$ with weaker priors on $N_{1}$ and $q$ (lognormal priors with $C V=5$ rather than $C V=2$ ). Inferences revealed insensitivity to these prior changes (not shown). We also checked whether the inferences were sensitive to the prior on the variance for the process and observation noise $\sigma^{2}$. 
A uniform prior distribution on the interval $(0,100)$ was tested for $\sigma^{2}$. Inferences were robust to these prior changes (not shown).

\section{Discussion}

\section{Strengths of the method}

We believe that we have offered three substantial new contributions. First, to our knowledge, this work provides the first BSSM approach of the De Lury depletion model including both process and observation errors and an estimation of a secondary recruitment pulse. Second, an extensive evaluation of the sensitivity of a Bayesian version of this model to alternative model forms and data configuration is provided. Third, the Bayesian hierarchical approach of McAllister et al. (2004) was extended by introducing an RW formulation to capture the systematic fluctuations of the time-series of initial abundance and catchability.

The SE approach provides an analysis of the performance of the method and an evaluation of the reliability of the outputs of the model. SSM was considered to be the favoured default estimation method. Both process and observation errors are likely to be found simultaneously in real datasets, and the SSM approach outperformed the OBS and PROC in the presence of both sources of error. The results allowed us to quantify how the performance of the estimation method depended on the strength of the depletion attributable to catch removal in the first time-step of each fishing season. The stronger the depletion, the less the bias and the uncertainty in the estimation. Also, when the data were sufficiently informative, the depletion model provided accurate estimates of both the principal and the secondary recruitment pulses. Yet, inferences appeared to be sensitive to mis-specification of $M$.

The methodology was applied to the data of the Moroccan fishery over the 21 fishing seasons between 1982 and 2002. The strong depletion rates observed in the Moroccan data offer an informative data configuration for model fitting. The model was designed to account for a secondary recruitment pulse spread over a few months during spring (as in Royer et al., 2002), in contrast to there being a single event (González-Yáñes et al., 2006). To ensure that all parameters could be estimated, we followed the approach of Royer et al. (2002) and considered the recruitment rhythm to be known. It was defined from previous studies (Balguerías et al., 2002) and the knowledge of local experts (AF, pers. obs.). Compared with Royer et al. (2002), who considered between-year variation in the recruitment rhythm, no data for between-year variation in $\lambda$ were available so we were constrained to consider it as constant across years. However, our results were insensitive to mis-specification of $\lambda$. Although this could have resulted from the fact that $R$ was low compared with the main recruitment, $N_{1}$, this outcome lends credibility to the estimates of the secondary recruitment pulse $R$.

Our analysis has provided estimates of hidden variables of important biological significance, such as annual recruitment (both primary and secondary) from 1982 to 2002, and estimates of the abundance each month. This, combined with catches, provides estimates of the annual exploitation rate. The estimates of high rates of exploitation presented here are consistent with previous findings (Faraj et al., 2006). The between-year variability of the recruitment during the 1982-2002 time-series also suggests a strong influence of the environment (Pierce et al., 2008), which merits future research.

The 21 depletion series in the Moroccan dataset were analysed using five alternative model structures of increasing complexity, ranging from model $M_{1}$ with all depletion series treated as independent to model $M_{5}$ where the values of $N_{1}$ and $q$ were treated as autocorrelated time-series within a hierarchical model. The BHM offers an advantageous modelling strategy to analyse such datasets with similar depletion patterns each year (McAllister et al., 2004). It organizes the transfer of information from years with informative data to years for which the data are less informative, improving the inference on non-informative years (Rivot and Prévost, 2002; McAllister et al., 2004; Rivot et al., 2008). We extended the hierarchical modelling framework by introducing RW on both $N_{1}$ and $q$. The model was the best compromise between quality of fit and complexity, and it allowed us to capture the smooth fluctuations in $N_{1}$ (and to a lesser extent in $q$ ). The smooth fluctuations in the values of $N_{1}$ may suggest a response to environmental variance.

\section{Caveats and future improvements}

Our model provides a framework for structuring further research and data collection.

Assumptions were made in the data preparation. In particular, total annual catches were disaggregated on a monthly time-step according to the monthly catch rhythm of the Moroccan industrial fleet. The assumption is realistic from 1982 to 1992, because the industrial fleet (Morocco and foreign countries) was the only one exploiting octopus then and operated throughout the year. The assumption is more questionable in the last 10 years (19922002), though, when artisanal and coastal operators entered the fishery. However, the industrial fleet still delivered $50-80 \%$ of the total landings. The data could be improved using catch and effort in monthly time-steps for the artisanal and coastal Moroccan fleets. However, the data were not available for the entire time-series, so we opted for a homogeneous approach for the whole time-series. Nevertheless, use of data from the artisanal fishery would be well worth considering in future.

The use of cpue as an index of abundance is also questionable, particularly because local effort could be positively correlated with local octopus abundance (Hilborn and Walters, 1992). As an improvement, standard statistical procedures such as generalized linear modelling could be used to extract the trend in abundance indices after having filtered other effects such as variability in size and/or horse power of trawlers (for examples, see Gascuel and Thiam, 1994; Gascuel et al., 2007). Scientific surveys are carried out in Morocco (Faraj and Bez, 2007), but because they are not spatially or temporally homogeneous in the entire time-series, we also decided not to use their output in this first methodological approach. One could rely on the flexibility of the estimation framework to integrate such sources of data in the model.

To reduce the number of parameters and ensure statistical identifiability, the ratio of process vs. observation errors was fixed. However, in the absence of any information on the magnitude of process variability and observation noise, the ratio of variance was set at 1 . This hypothesis is worth investigating further, though. Inferences on SSMs have seldom proved to be sensitive to the value of this ratio (Kimura et al., 1996; Schnute and Kronlund, 2002; Punt, 2003), but SSM offers the advantages of independently integrating process and observation error, and more research is needed to help formulate more informative priors on the variances.

The SE approach and the sensitivity analysis showed that inferences are highly sensitive to assumptions on $M$. Little is known about the natural mortality of octopus in the wild. The 
value of $M$ was fixed at 0.1 , which led to realistic mortality rates of $\sim 45,70$, and $83 \%$ after 6,12 , and 18 months, respectively. The results showed that using $M=0.2$ led to higher estimates of abundance. However, the value of $M=0.2$ tested here for illustration has little biological significance, because it leads to an unrealistic mortality rate of $\sim 70 \%$ after only 6 months. Hence, we believe that the illustrative sensitivity analysis overappraised the uncertainty of our results as a consequence of uncertainty about the value of $M$. It might be of interest to propose an alternative modelling strategy that better mimics true natural mortality processes. The octopus is a semelparous animal, so setting a constant rate of natural mortality appears to be a crude assumption. The model we proposed pools the two annual cohorts in the same dynamic equation, preventing the use of age-dependent mortality. For in-season stock assessment of the squid off the Falkland Islands, Agnew et al. (1998) used a multiple cohort approach, treating data as representing two distinct cohorts with their own dynamic equations. That approach allows definition of age-dependent rates of mortality. Building a two-cohort depletion model with age-dependent natural mortality (low mortality during the first months, increasing to a plateau in later months when reproduction starts) should be considered.

Some fixed hypotheses have been formulated about the parameter $q$. In particular, $q$ has been considered to be constant between months within a fishing season. However, catchability at the start of the period is likely to be higher than at the end of the period owing to changes in the behaviour of the animals at the onset of reproduction (AF, pers. obs.). However, a modelling strategy of varying $q$ should be used with caution because it would increase the number of unknowns to be estimated and therefore the risk of non-identifiability.

\section{Time-series of recruitment for understanding the influence of the environment}

The role of upwelling in the bottom-up control of marine ecosystems is well established: strengthening of the trade wind generates upwelling of deep, nutrient-rich water to the surface, producing optimal environmental conditions for plankton growth (Cury and Roy, 1989; Bakun, 1990; Cushing, 1990). Pelagic larvae of octopus should benefit from retention processes in enriched areas (Demarcq and Faure, 2000; Faure et al., 2000; Otero et al., 2008). Good survival of pelagic larvae should support high levels of recruitment (Rodhouse et al., 1992; Bakun and Csirke, 1998; Waluda et al., 1999). Previous studies have demonstrated the effects of sea temperature and retention processes on recruitment fluctuations in cephalopods: Loligo gahi in the South Atlantic (Agnew et al., 2000), Loligo forbesi in the English Channel (Robin and Denis, 1999), O. vulgaris along the Galician coast (Otero et al., 2008) and on the Saharan Bank (Demarcq and Faure, 2000; Balguerías et al., 2002). The recruitment index provided here could be used further to investigate the influence of the upwelling regime on interannual variations in octopus population dynamics in the context of both medium- and long-term changes (Bakun, 1990; Santos et al., 2005). However, there are clear distinctions between the spatial patterns of spawning and recruitment of the Moroccan stocks (Faraj and Bez, 2007), and more research is needed to integrate this spatial dimension into our Bayesian estimation approach.

\section{Implications for management}

Finally, the BSSM approach to stock assessment with a depletion model provides an integrated framework for data integration and prediction that accounts for multiple sources of uncertainty. It could therefore be applied to develop a predictive approach for decision-making and risk analysis. A depletion model applied on a weekly time-step is under development for in-season stock assessment and operational management of the Moroccan octopus fishery (Faraj et al., 2006). The hierarchical BSSM approach developed here could be adapted to that weekly timestep if desired.

\section{Acknowledgements}

The study was carried out with financial support from the ISTAM project (EU FP7 contract 022774). The paper was completed thanks to scientific collaboration with the laboratory of Approach and Methodology of the INRH (National Institute of Fisheries Sciences) of Casablanca. We especially thank Khalid Manchih for his help, and Emmanuel Chassot, Didier Gascuel, Sylvain Bonhommeau, and Brahim O. Mohamed Tfeil for useful discussions. Anonymous reviewers provided helpful comments that greatly improved an earlier version of the manuscript.

\section{References}

Agnew, D. J., Baranowski, R., Beddington, J. R., Des Clers, S., and Nolan, C. P. 1998. Approaches to assessing stocks of Loligo gahi around the Falkland Islands. Fisheries Research, 35: 155-169.

Agnew, D. J., Hill, S., and Beddington, J. R. 2000. Predicting the recruitment strength of an annual squid stock: Loligo gahi around the Falkland Islands. Canadian Journal of Fisheries and Aquatic Sciences, 57: 2479-2487.

Bakun, A. 1990. Global climate change and intensification of coastal ocean upwelling. Science, 247: 198-201.

Bakun, A., and Csirke, J. 1998. Environmental processes and recruitment variability. In Squid Recruitment Dynamics. The Genus Illex as a Model, the Commercial Illex Species and Influences on Variability, pp. 105-120. Ed. by P. G. Rodhouse, E. G. Dawe, and R. K. O'Dor. FAO Fisheries Technical Paper, 376.

Balguerías, E., Hernández-González, C., and Perales-Raya, C. 2002. On the identity of Octopus vulgaris (Cuvier 1797) stocks in the Saharan Bank (Northwest Africa) and their spatio-temporal variations in abundance in relation to some environmental factors. Bulletin of Marine Science, 71: 147-163.

Balguerías, E., Quintero, M. E., and Hernández-Gonzáles, C. L. 2000. The origin of the Saharan Bank cephalopod fishery. ICES Journal of Marine Science, 57: 15-23.

Beddington, J. R., Rosenberg, A. A., Crombie, J. A., and Kirkwood, G. P. 1990. Stock assessment and the provision of management advice for the shortfin squid fishery in Falkland Islands waters. Fisheries Research, 8: 351-365.

Buckland, S. T., Newman, K. B., Thomas, L., and Koesters, N. B. 2004. State-space models for the dynamics of wild animal populations. Ecological Modelling, 171: 157-175.

Caddy, J., and Rodhouse, P. G. 1998. Comparison of recent trends in cephalopod and groundfish landings: an indicator of widespread ecological change in global fisheries. Reviews in Fish Biology and Fisheries, 8: 431-444.

Clark, J. S. 2005. Why environmental scientists are becoming Bayesians. Ecology Letters, 8: 2-14.

Cury, P., and Roy, C. 1989. Optimal environmental window and pelagic fish recruitment success in upwelling areas. Canadian Journal of Fisheries and Aquatic Sciences, 46: 670-680. 
Cushing, D. H. 1990. Plankton production and year-class strength in fish populations: an update of the match mismatch hypothesis. Advances in Marine Biology, 26: 249-293.

De Lury, D. B. 1947. On the estimation of biological populations. Biometrics, 3: 145-167.

Demarcq, H., and Faure, V. 2000. Coastal upwelling and associated retention indices derived from satellite SST. Application to Octopus vulgaris recruitment. Oceanologica Acta, 23: 391-408.

Ellison, A. M. 2004. Bayesian inference in ecology. Ecology Letters, 7: $509-520$.

FAO. 2006. Report of the FAO/CECAF Working Group on the Assessment of Demersal Resources-Subgroup North Saly, Senegal, 14-23 September 2004. Series 06/68.

Faraj, A., Azeguagh, I., Bensbai, J., Boumaaz, A., Manchih, K., Najd, A., Hamdi, H., et al. 2006. Rapport de la $1^{\text {ère }}$ Réunion du Groupe de Travail "Evaluation de stock des céphalopodes". INRH, Casablanca. 31 pp.

Faraj, A., and Bez, N. 2007. Spatial considerations for the Dakhla stock of Octopus vulgaris: indicators, patterns, and fisheries interactions. ICES Journal of Marine Science, 64: 1820-1828.

Faure, V., Inejih, C. A., Demarcq, H., and Cury, P. 2000. The importance of retention processes in upwelling areas for recruitment of Octopus vulgaris: the example of the Arguin Bank (Mauritania). Fisheries Oceanography, 9: 343-355.

Gascuel, D., Labrosse, P., Taleb Sidi, M. O., and Guénette, S. 2007. Decline of demersal resources in North-West Africa: an analysis of Mauritanian trawl-survey data over the past 25 years. African Journal of Marine Science, 29: 331-345.

Gascuel, D., and Thiam, M. 1994. Evolution de l'abondance des ressources démersales sénégalaises: estimation par modélisation linéaire des PUE. In L'évaluation des Ressources Exploitables par la Pêche Artisanale sénégalaise, Symposium Dakar, 8-13 février 1993, pp. 191-213. Ed. by M. Barry-Gérard, T. Diouf, and A. Fonteneau. ORSTOM Editions, collection colloques et séminaires, Tome 2. 424 pp.

Gelman, A., Carlin, J. B., Stern, H., and Rubin, D. B. 2004. Bayesian Data Analysis, 2nd edn. Chapman and Hall, London. 668 pp.

Gonzalez Cano, J., and Restrepo, V. R. 2001. Stock assessment methods. Part 3. FAO Fisheries Report, 619: 345-356.

González-Yáñez, A., Millán, R. P., Léon, M. E., Cruz-Font, L., and Wolff, M. 2006. Modified Delury depletion model applied to spiny lobster, Panulirus argus (Latreille, 1804) stock, in the southwest of the Cuban shelf. Fisheries Research, 79: 155-161.

Halley, J., and Inchausti, P. 2002. Lognormality in ecological time series. Oikos, 99: 518-530.

Hammond, T. R., and Trenkel, V. 2005. Censored catch data in fisheries stock assessment. ICES Journal of Marine Science, 62: $1118-1130$.

Harwood, J., and Stokes, K. 2003. Coping with uncertainty in ecological advice: lessons from fisheries. Trends in Ecology and Evolution, 18: 617-622.

Hilborn, R., and Lierman, M. 1998. Standing on the shoulders of giants: learning from experience in fisheries. Reviews in Fish Biology and Fisheries, 8: 273-283.

Hilborn, R., and Walters, C. J. 1992. Quantitative Fisheries Stock Assessment. Choice, Dynamics and Uncertainty. Chapman and Hall, London.

Kimura, D. K., Balsiger, J. W., and Ito, D. H. 1996. Kalman filtering the delay-difference equation: practical approaches and simulations. Fishery Bulletin US, 94: 678-691.

Leslie, P. H., and Davis, D. H. S. 1939. An attempt to determine the absolute number of rats on a given area. Journal of Animal Ecology, 8: 94-113.

Lunn, D. J., Spiegelhalter, D., Thomas, A., and Best, N. 2009. The BUGS project: evolution, critique and future directions. Statistics in Medicine, 28: 3049-3067.
Magnusson, A., and Hilborn, R. 2007. What makes fisheries data informative? Fish and Fisheries, 8: 337-358.

McAllister, M. K., Hill, S. L., Agnew, D. J., Kirkwood, G. P., and Beddington, J. R. 2004. A Bayesian hierarchical formulation of the De Lury stock assessment model for abundance estimation of Falkland Islands' squid (Loligo gahi). Canadian Journal of Fisheries and Aquatic Sciences, 61: 1048-1059.

McAllister, M. K., and Kirkwood, G. P. 1998. Bayesian stock assessment: a review and example application using the logistic model. ICES Journal of Marine Science, 55: 1031-1060.

Meyer, R., and Millar, R. B. 1999. BUGS in Bayesian stock assessments. Canadian Journal of Fisheries and Aquatic Sciences, 56: 1078-1086.

Millar, R. B. 2002. Reference priors for Bayesian fisheries models. Canadian Journal of Fisheries and Aquatic Sciences, 59: 1492-1502.

Millar, R. B., and Meyer, R. 2000. Non linear state-space modelling of fisheries biomass dynamics by using Metropolis-Hastings within-Gibbs sampling. Journal of the Royal Statistical Society, 49: $327-342$.

Otero, J., Alvarez-Salgado, X. A., González, A., Miranda, A., Groom, S. B., Cabanas, J. M., Casas, G., et al. 2008. Bottom-up control of common octopus, Octopus vulgaris, in the Galician upwelling system, northeast Atlantic Ocean. Marine Ecology Progress Series, 362: 181-192.

Payne, A. G., Agnew, D. J., and Pierce, G. J. 2006. Foreword: trends and assessment of cephalopod fisheries. Fisheries Research, 78: 1-3.

Pierce, G. J., and Guerra, A. 1994. Stock assessment methods used for cephalopod fisheries. Fisheries Research, 21: 255-285.

Pierce, G. J., Valavanis, V. D., Guerra, A., Jereb, P., Orsi-Relini, L., Bellido, J. M., Katara, I., et al. 2008. A review of cephalopodenvironment interactions in European seas. Hydrobiologia, 612: $49-70$.

Pope, J. G. 1972. An investigation of the accuracy of virtual population analysis using cohort analysis. ICNAF Research Bulletin, 9: 65-74.

Punt, A. E. 2003. Extending production models to include process error in the population dynamics. Canadian Journal of Fisheries and Aquatic Sciences, 60: 1217-1228.

Punt, A. E., and Hilborn, R. 1997. Fisheries stock assessment and decision analysis: the Bayesian approach. Reviews in Fish Biology and Fisheries, 7: 35-63.

Rivot, E., and Prévost, E. 2002. Hierarchical Bayesian analysis of capture-mark-recapture data. Canadian Journal of Fisheries and Aquatic Sciences, 59: 1768-1784.

Rivot, E., Prévost, E., Cuzol, A., Parent, E., and Baglinière, J. L. 2008. Hierarchical Bayesian modelling with habitat and time covariates for estimating riverine fish population size by successive removal method. Canadian Journal of Fisheries and Aquatic Sciences, 65: $117-133$.

Rivot, E., Prévost, E., Parent, E., and Baglinière, J. L. 2004. A Bayesian state-space modelling framework for fitting a salmon stagestructured population dynamic model to multiple time series of field data. Ecological Modelling, 179: 463-485.

Roa-Ureta, R., and Arkhipkin, A. I. 2007. Short-term stock assessment of Loligo gahi at the Falkland Islands: sequential use of stochastic biomass projection and stock depletion models. ICES Journal of Marine Science, 64: 3-17.

Robin, J-P., and Denis, V. 1999. Squid stock fluctuations and water temperature: temporal analysis of English Channel Loliginidae. Journal of Applied Ecology, 36: 101-110.

Rodhouse, P. G., Symon, C., and Hatfield, E. M. C. 1992. Early life cycle of cephalopods in relation to the major oceanographic features of the southwest Atlantic Ocean. Marine Ecology Progress Series, 89: 183-195.

Rosenberg, A. A., Kirkwood, G. P., Crombie, J. A., and Beddington, J. R. 1990. The assessment of stocks of annual squid species. Fisheries Research, 8: 335-350. 
Royer, J., Périès, P., and Robin, P. 2002. Stock assessments of English Channel loliginid squids: updated depletion methods and new analytical methods. ICES Journal of Marine Science, 59: 445-457.

Santos, A. M., Kazmin, A. S., and Peliz, A. 2005. Decadal changes in the Canary upwelling system as revealed by satellite observations: their impact on productivity. Journal of Marine Research, 63: 359-379.

Schnute, J. T., and Kronlund, A. R. 2002. Estimating salmon stockrecruitment relationships from catches and escapement data. Canadian Journal of Fisheries and Aquatic Sciences, 59: 433-449.

Spiegelhalter, D. J., Best, N. G., Carlin, B. P., and Van der Linde, A. 2002. Bayesian measures of model complexity and fit. Journal of the Royal Statistical Society, Series B, 64: 1-34.

Swain, D. P., Jonsen, I. D., Simon, J. E., and Myers, R. 2009. Assessing threats to species at risk using stage-structured state-space models: mortality trends in skate populations. Ecological Applications, 19: $1347-1364$

Tang, S., and Heron, E. A. 2008. Bayesian inference for a stochastic logistic model with switching points. Ecological Modelling, 219: 153-169.

Thomas, A., Spiegelhalter, D. J., and Gilks, W. R. 1992. BUGS: a program to perform Bayesian inferences using Gibbs sampling. In Bayesian Statistics, 4, pp. 837-842. Ed. by J. A. Bernardo, J. O. Berger, A. P. David, and A. F. M. Smith. Clarendon Press, Oxford, UK.
Thomas, L., Buckland, S. T., Newman, K. B., and Harwood, J. 2005. A unified framework for modelling wildlife population dynamics. Australian and New Zealand Journal of Statistics, 47: 19-34.

Trenkel, V. M. 2008. A two-stage biomass random effect model for stock assessment without catches: what can be estimated using only biomass survey indices? Canadian Journal of Fisheries and Aquatic Sciences, 65: 1024-1035.

Walters, C., and Ludwig, D. 1994. Calculation of Bayes posterior probability distributions for key population parameters. Canadian Journal of Fisheries and Aquatic Sciences, 51: 713-722.

Waluda, C., Trathan, P., and Rodhouse, P. 1999. Influence of oceanographic variability on recruitment in the Illex argentinus (Cephalopoda: Ommastrephidae) fishery in the South Atlantic. Marine Ecology Progress Series, 183: 159-167.

Young, I. A. G., Pierce, G. J., Daly, H. I., Santos, M. B., Key, L. N., Bailey, N., Robin, J-P., et al. 2004. Application of depletion methods to estimate stock size in the squid Loligo forbesi in Scottish waters (UK). Fisheries Research, 69: 211-227.

Zhang, Z., Lessard, J., and Campbell, A. 2009. Use of Bayesian hierarchical models to estimate northern abalone, Haliotis kamtschatkana, growth parameters from tag-recapture data. Fisheries Research, 95: 289-295.

doi:10.1093/icesjms/fsq020 